\title{
Bogdanov-Takens Bifurcation Points and Šil'nikov Homoclinicity in a Simple Power-System Model of Voltage Collapse
}

\author{
C. J. Budd and J. P. Wilson
}

\begin{abstract}
The bifurcation structure of a simple power-system model is investigated, with respect to changes to both the real and reative loads. Numerical methods for this bifurcation analysis are presented and discussed. The model is shown to have a Bogdanov-Takens bifurcation point and hence homoclinic orbits; these orbits can be of Šil'nikov type with many coexisting periodic solutions. We may use the bifurcation calculations to divide the two-parameter plane into a number of regions, for which there are qualitatively different dynamics. We classify and further investigate the dynamical behavior in each of these regions, using a Monte Carlo method to investigate basins of attraction of various stable states. We then show how this classification can be used to denote each regions as either safe or unsafe with respect to the likelihood of voltage collapse.
\end{abstract}

Index Terms-Basinofattraction, bifurcation, BogdanovTakens, continuation, dynamical system, homoclinic orbit, Monte Carlo, power system, shilnikov, Takens-Bogdanov, voltage collapse.

\section{INTRODUCTION}

$\mathbf{E}$ LECTRICAL generation and distribution networks form some of the largest and most complex manmade systems. While many aspects of power systems and their individual components are well understood and have been researched in great depth, other phenomena evident in these systems remain relatively unexplained. Economic and other pressures mean that power systems are now being run ever closer to their operating limits. This has lead to an increasing number of "voltage collapse" incidents [1]-[3], resulting in system breakdown on a large scale. In such behavior we see the voltage dropping to zero in a very short time.

Greater awareness of modern mathematical methods, more robust computational algorithms and increased computer power, have all led to power systems researchers using techniques not previously considered practical given the size of "real-life" systems. Over the last few years, a number of researchers have discussed the application of dynamical systems and bifurcation theory to power networks. In particular voltage collapse has been related to the destabilization of fixed operating points at

Manuscript received January 19, 2000; revised August 30, 2000. This paper was recommended by Associate Editor H. S. H. Chung.

The authors are with the University of Bath, Bath, BA2 7AY, U.K. (e-mail: cjb@maths.bath.ac.uk; jon@netsw.co.uk).

Publisher Item Identifier S 1057-7122(02)04726-8. saddle-node and Hopf bifurcation points and to the erosion of the basins of attraction of stable fixed points.

In this paper, we consider a reduced power-system model, previously investigated by a number of researchers and argue that the dynamical behavior of this model can be best understood in terms of a Bogdanov-Takens (BT) bifurcation point and an associated (global) Šil'nikov bifurcation. This model comprises three buses modeling two generators supplying power to a load. Whilst highly simplified from a stand-point of electrical engineering, this model is still very rich dynamically and exhibits many of the features of voltage-collapse that we are interested in.

We show some unobserved behavior in this system and in particular demonstrate that the parameter regimes which might be considered stable may be overly optimistic. In particular we identify the existence of a BT bifurcation point with nearby Šil'nikov-type homoclinic orbits. The BT-point acts as a organizing centre in the bifurcation structure of the model and plays a significant role in helping to understand the overall mechanism of voltage collapse through the destabilization of fixed points and the erosion of basin boundaries. BT-points will arise naturally in much larger systems as operating parameters change and hence we consider the observed dynamics of the 3-bus model to be a fairly general explanation for much of the dynamics associated with voltage collapse. Because of the importance of the BT-point we consider in detail techniques for calculating it both for the reduced system and also for more complex systems.

The layout of the remainder of this paper is as follows. In Section II we introduce the model used and briefly discuss some of the dynamics associated with voltage collapse. In Section III we introduce some of the basic mathematics we will use, in particular, bifurcation theory and the BT bifurcation. We describe the method of numerical path following used and discuss basins of attraction and their numerical approximation. In Section IV we demonstrate the existence of saddle-node and Hopf bifurcations, and show how these lead to a BT-point and a closely related Sil'nikov bifurcation. In Section V we relate the existence of the various bifurcation points to the overall dynamics observed in the model. In particular, we study the basins of attraction of various attracting states and differentiate between those solutions which remain bounded and those which exhibit voltage collapse. In Section VI we draw some conclusions from this work. In the Appendix we look at the computation of BT-points in both the model problem and higher dimensional systems. 


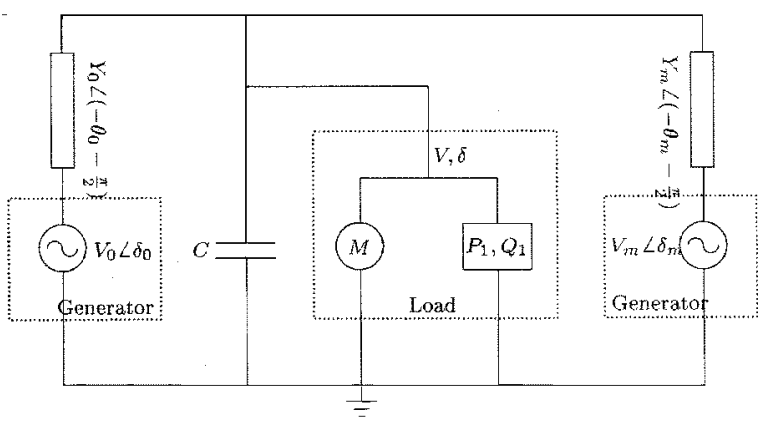

Fig. 1. Circuit diagram for the 3-Bus model.

\section{3-Bus MODEL DESCRIPTION}

We consider a 3-bus model introduced in [4], [5] and further investigated in [6]-[11]. Whilst being simple, it has the same basic dynamical structure of more realistic power-system models. The model comprises two generator buses and a load bus connected according to the circuit diagram in Fig. 1.

One generator is modeled by a simple swing equation model and the other is a slack bus acting as an infinite power source at fixed voltage $V_{0} \angle \delta_{0}$.

The real and reactive loads at the third bus comprise fixed real and reactive loads $P_{1}$ and $Q_{1}$, in parallel with an empirical model of induction motor load suggested by [1]. We note that in general, many power-system load models involve "static" relations between the real and reactive loads $P, Q$, and the supplied voltage $V$ and relative phase angle $\delta_{m}$. There is no reason to presuppose that the load will be independent of the derivatives of $V$ and $\delta_{m}$. If the dynamical behavior of power systems is to be properly investigated it is necessary to to use models of the system components which involve time derivatives of the system states (i.e., models which are truly dynamic in the mathematical sense).

Normally, the application of Kirchoff's laws to calculate the power flow through the network would give an algebraic constraint on the system leading to a differential-algebraic system. However, with this simple network we substitute $P_{e}, P_{l}$ and $Q_{l}$ into the dynamical part of the model and write the entire system as a four-dimensional ordinary differential equation

$$
\begin{aligned}
\dot{\delta}_{m}= & \omega_{m} \\
\dot{\omega}_{m}= & \frac{1}{M}\left(-d_{m} \omega_{m}+P_{m}-P_{e}\right) \\
\dot{\delta}= & \frac{1}{K_{q \omega}}\left(-K_{q v} V-K_{q v^{2}} V^{2}+Q_{l}-Q_{0}-Q_{1}\right) \\
\dot{V}= & \frac{1}{T K_{q \omega} K_{p v}}\left(K_{p \omega} K_{q v^{2}} V^{2}+\left(K_{p \omega} K_{q v}-K_{q \omega} K_{p v}\right) V\right. \\
& \left.+K_{p \omega}\left(Q_{0}+Q_{1}-Q_{l}\right)-K_{q \omega}\left(P_{0}+P_{1}-P_{l}\right)\right) .
\end{aligned}
$$

where $P_{e}, P_{l}$ and $Q_{l}$ are given by

$$
\begin{aligned}
P_{l}= & -V_{0}^{\prime} V Y_{0}^{\prime} \sin \left(\delta+\theta_{0}^{\prime}\right)-V_{m} V Y_{m} \sin \left(\delta-\delta_{m}+\theta_{m}\right) \\
& +\left(Y_{0}^{\prime} \sin \left(\theta_{0}^{\prime}\right)+Y_{m} \sin \left(\theta_{m}\right)\right) V^{2} \\
Q_{l}= & -V_{0}^{\prime} V Y_{0}^{\prime} \cos \left(\delta+\theta_{0}^{\prime}\right)-V_{m} V Y_{m} \cos \left(\delta-\delta_{m}+\theta_{m}\right) \\
& +\left(Y_{0}^{\prime} \cos \left(\theta_{0}^{\prime}\right)+Y_{m} \cos \left(\theta_{m}\right)\right) V^{2} \\
P_{e}= & -V_{m} V Y_{m} \sin \left(\delta-\delta_{m}-\theta_{m}\right)-V_{m}^{2} Y_{m} \sin \left(\theta_{m}\right) .
\end{aligned}
$$

We use the following system parameters, which are all dimensionless, apart from the angles which are in radians. Generator parameters: $M=0.3, D=0.05, P_{m}=1.0$. Load parameters: $K_{p \omega}=0.4, K_{p v}=0.3, K_{q \omega}=-0.03, K_{q v}=-2.8$, $K_{q v^{2}}=2.1, T=8.5, P_{0}=0.6, Q_{0}=1.3$. Network parameters: $V_{m}=1.0, Y_{m}=5.0, \theta_{m}=-5 \pi / 180, V_{0}^{\prime}=2.5, Y_{0}^{\prime}=$ $8.0, \theta_{0}^{\prime}=-12 \pi / 180$.

In the load, the constant parts of the real and reactive power demands $\left(P_{1}\right.$ and $\left.Q_{1}\right)$ are parameters which could in principle be varied in an experiment. In principle we could also vary the system damping and inductance or other parameters, but we shall not consider doing this here. By varying $P_{1}$ and $Q_{1}$ we identify certain types of dynamics of (1) and the transitions between them.

\section{BIFURCATIONS AND NumerICAL METHODS}

\section{A. Basic Definitions}

The 3-bus model (1) is an example of a general class of parameterised autonomous dynamical systems defined by the solution to the $n$-dimensional ODE

$$
\dot{x}=f(x, \lambda)
$$

with system state variables $x \in \Omega \subseteq \mathbb{R}^{n}$, parameters $\lambda \in \mathbb{R}^{m}$ and $f: \Omega \times \mathbb{R}^{m} \rightarrow \mathbb{R}^{n}$ a smooth function. For the 3-bus model $n=4, x=\left(\delta_{m}, \omega_{m}, \delta, V\right)$ and $\lambda=\left(P_{1}, Q_{1}\right)$.

1) Bifurcations: The overall dynamics of (3) can be partially understood in terms of the bifurcations that occur as one of the components in the parameter $\lambda$ changes. At such a bifurcation point the flow mapping ${ }^{1}$ defined by (3) undergoes a qualitative change [12]. Bifurcation can be classified as either global or local; static or dynamic. All are observed in the 3-bus system.

Local static bifurcations of fixed points may occur when the system Jacobian matrix $f_{x}$ has a simple zero eigenvalue, such as the saddle-node bifurcation when two fixed points coalesce. When $f_{x}$ has a pair of purely imaginary eigenvalues, the system may undergo Hopf bifurcation in which the fixed point becomes unstable and is replaced by a nearby periodic orbit satisfying $x(t)=x(t+T)$ for some $T$. This is an example of a local, dynamic bifurcation. From a power engineering perspective, since oscillatory behavior is undesirable, we can consider Hopf bifurcations to be generally destabilising, even though they may not lead directly to voltage collapse. Both of these forms of bifurcation are observed repeatedly in the 3-bus model.

It is possible for periodic orbits (which may or may not have been created in a Hopf bifurcation) to themselves undergo changes at local bifurcations in the Poincaré map associated with the orbit. In particular, we see period doubling bifurcations when an eigenvalue of this map equals -1 and cyclic fold bifurcations when an eigenvalue equals 1 .

We also observe global bifurcations leading to more dramatic changes in the dynamical behavior of the system. An example of such a global bifurcation occurs at a homoclinic bifurcation. A periodic orbit may grow as a parameter changes so that it intersects an unstable fixed point to give an infinite period homoclinic orbit $\{x(t): t \in[-\infty, \infty]\}$ for which there is a point

\footnotetext{
${ }^{1}$ The parameterised function mapping all possible states of the system at time $t$ to their states at time $(t+\tau)$.
} 
$x^{*}$ with $x(t) \rightarrow x^{*}$ as $t \rightarrow \pm \infty$. This bifurcation is global as it cannot be detected merely by local analysis alone (for instance by monitoring eigenvalues of $f_{x}$ at $x^{*}$ ).

A more detailed mathematical description of all these concepts can be found in texts such as [13]. It should be emphasized that bifurcations do not give the whole picture for a phenomenon such as voltage collapse. Here, a very important role is played by the basins of attraction of the relevant attractors, such as the fixed points, periodic and chaotic attractors.

\section{B. A Brief Description of the System Dynamics}

The system represented by the 3-bus model (1) ideally operates in a fixed, stable, steady state. If such a state is unique and globally attracting — or at least has a large basin of attraction-then we can consider the overall system to be operating safely. Large deviations of initial conditions from the steady state (caused, for example, by a power line failure or some other fault) will not prevent the system from returning to its stable operating conditions. For a wide class of parameter values, it is known [14, Ch. 14], that the power systems with networks of this type will generally have two (or indeed several) fixed operating points where all time derivatives vanish. One of these points is a stable attractor whereas the other is an unstable saddle point.

As either $P_{1}$ or $Q_{1}$ are varied then the fixed points also vary, typically meeting at a saddle-node bifurcation point at which we have maximum power transfer. Past this point, there are no fixed operating points and typically the power system is unstable with an immediate collapse of the voltage. Such voltage collapse corresponds mathematically to a solution of (1) which is unbounded in finite time. Voltage collapse can occur either when there is no attracting bounded solution or when an initial state (for instance caused by a transient due to a local power failure) lies outside the basin of attraction of such a bounded state.

The computation of saddle-node bifurcations is important, as it defines the maximum possible region of safe operation. Various attempts have been made to obtain estimates for these points in more general systems. However, as we demonstrate below, voltage collapse can easily occur prior to saddle-node bifurcation, due to complex behavior arising from coexisting periodic, steady-state and chaotic solutions.

\section{Numerical Path Following Methods}

The bifurcations described above occur as a parameter (say the reactive power $Q_{1}$ ) is varied. The value of $Q_{1}$ at which the bifurcation occurs depends in turn on other parameters such as $P_{1}$. To investigate the system fully it is important to have a numerical method which can both look at the varying dynamics as one parameter is varied and can also follow paths of bifurcation points in a second parameter, such that if a (saddle-node or Hopf) bifurcation occurs at $Q_{1}^{\text {Bif }}$ then the value of $Q_{1}^{\text {Bif }}$ can be determined as a function of the second parameter (such as $P_{1}$ ).

A useful tool for such a numerical investigation is Keller's pseudo-arc length continuation method. Given a fixed point $x_{0}$ of our system at a particular parameter value $Q_{1}^{(0)}$, the implicit function theorem [15] implies the existence of a locus of such fixed points in parameter and phase space. We wish to approximate this locus by finding a new fixed point $x_{1}$ for some $Q_{1}^{(1)}$ in a neighborhood of $Q_{1}^{(0)}$. We could choose $Q_{1}^{(1)}$ and then find a root of (3) using a Newton-type method; however this "parameter" continuation method may fail if $Q_{1}^{(1)}$ is at or near a turning point of the solution locus. Instead, if we calculate the tangent to the solution locus at $\left(x_{0}, Q_{1}^{(0)}\right)$ (the "pseudo-arc"), take a point $\left(x, Q_{1}\right)$ some distance along it, and then iterate to find a new fixed point $\left(x_{1}, Q_{1}^{(1)}\right)$, we will have a highly stable algorithm. This is the basic method implemented in numerical bifurcation packages such as AUTO [16] and in the authors own code. The pseudo-arc continuation method has been applied to power engineering problems but is not always known as such - for a useful survey of the use of such methods in power systems see [17]. The method may be extended to compute the loci of other dynamically interesting solutions such as loci of periodic orbits.

The detection of both saddle-node and Hopf bifurcation points is then determined by monitoring the eigenvalues of the linearization of the system during the course of the computation. To calculate the loci of such bifurcation points in 2-parameter space, a suitable extended system is formed, the roots of which define points on the locus. The continuation algorithm may then be applied to the extended system. In practice, the size of these extended systems may be relatively large, so reformulations of the problem and efficient solution methods must be considered. For instance, see [18], [19].

\section{BT Points}

The algorithm above accurately computes paths of Hopf and saddle node bifurcations. In particular it can identify special values of the second parameter at which these two paths intersect. Such a point is generally referred to as a BT point. BT points have been shown to play an important role in 'organizing' the dynamics of a variety of physical systems of high dimension, for example [20]-[22]. A similar organization is seen in the 3-bus model.

A BT-point is a co-dimension two bifurcation point; where a fixed point of the system has a Jacobian matrix $f_{x}$ with a zero eigenvalue of algebraic multiplicity 2 but of geometric multiplicity 1 [13]. Subject to satisfaction of transversality conditions and absence of certain symmetries in the system [13] this point occurs when there is a tangential intersection, in the 2-parameter plane, of a path of folds and a path of Hopf-bifurcations. An algorithm for the calculation of these points in a larger system is given in the Appendix.

For parameter values close to that of the BT-point the dynamics of the system can be reduced to that of a much simpler system given by one of the following normal forms:

$$
\begin{aligned}
& \dot{x}=y \\
& \dot{y}=\nu_{1}+\nu_{2} x+x^{2} \pm x y .
\end{aligned}
$$

This means that near a BT point, the dynamics of the system (3) are governed by the solution of (4) on a two-dimensional (2-D) center-manifold [23] of the larger system. The dynamics on this manifold, in particular the behavior of the periodic and homoclinic orbits, completely describes the local behavior of the original system. This remains true even for systems of much higher dimension than considered here and it is for this reason that we believe that an analysis of the BT-point for the simple 
3-bus model is extendable and will give insight into the general behavior of more complex problems.

A more detailed analysis [13] of the normal form (4) shows that a path of homoclinic orbits also emanates from such a BT-point. That is along a curve in the $\left(P_{1}, Q_{1}\right)$ space there is a homoclinic orbit. At the BT-point this curve is also tangential to the loci of the saddle-node and Hopf points. As we shall demonstrate, the existence of this homoclinic orbit has a profound effect on the overall stability of the 3-bus system. A further discussion of the numerical detection of BT-points is given in the Appendix.

\section{E. Branch Switching at BT-Point}

Starting from a BT-point, continuation methods can be used to determine paths of folds, Hopf-bifurcations, and homoclinic orbits in the $\left(P_{1}, Q_{1}\right)$ parameter space. However, to do this we will need to calculate an initial point on each of the paths.

For the computation of a paths of folds and Hopf points emanating from the BT-point, the initial step in the continuation algorithm will converge provided we do not attempt to step too far from the BT-point. It is important not to choose a value too small, as the Jacobian of the defining system for the bifurcation point locus may be singular [20].

Details of a method to start the calculation of the path of homoclinic orbits emanating from the BT-point are given in [24].

This gives us a methodology for a more complete detection of the bifurcation structure. First locate a fixed point of the system (e.g., a stable operating point); perform continuation on this fixed point and detect a saddle-node bifurcation. Compute a path of saddle-node bifurcations and detect a BT-point. Finally, compute paths of Hopf and homoclinic bifurcations emanating from the BT-point.

\section{F. Basin Erosion}

When considering the possibility of a systems such as the 3-bus model (1) starting from a general initial condition and potentially exhibiting voltage collapse, we are interested in the basins of attraction for the attractors of that system. These are the sets of initial state values for which the corresponding trajectories have the property that they converge to the stable fixed point, or to a stable periodic orbit, or to a chaotic orbit (and are thus bounded). These basins are strongly parameter dependent. It is well known that even for very simple dynamical systems the boundaries of these basins may be highly complicated, even fractal [25]. For calculations of safety margins and for avoiding voltage collapse we must find how these basins change in size and/or form as parameters change, particularly as we approach bifurcation points.

From an engineering perspective, the calculation of these basins will give information as to what perturbations away from the stable fixed point are permissable (i.e., the trajectory returns to the desired operating point) and which are inpermissable (i.e., convergence to some other attractor, or voltage collapse).

In practice, we cannot investigate the whole of phase space, and so we must pick a bounded subset to use as our region of investigation for studies of basins of attraction.

1) Review of Methods-Lyapunov Functions, Cell Mapping and Manifold Estimation: Various methods exist for estimating basins of attraction. A widely used method is the construction of a Lyapunov (or "energy") function for the system; trajectories starting from points where this function is concave will converge to the stable point about which the function is constructed. Hence the boundary of the region of concavity of the Lyapunov function is an estimate of the boundary of the basin of attraction.

There are two reasons why this approach has not been followed here. Firstly, although such functions have been constructed successfully for a wide class of power-system models [26]-[28], it is not clear how to construct a Lyapunov function for the four-dimensional system (1) with the dynamical load model of the type we consider necessary for proper understanding of the voltage collapse problem.

Secondly, and more importantly, the Lyapunov function method will only give a subset of the basin of attraction. There may be points outside the region of concavity which have trajectories that converge to the attractor. If the basin boundary is highly structured (e.g., fractal, or if there are multiple attractors) the Lyapunov estimate may be very bad indeed. It is also not known precisely whether the Lyapunov function gives a good indication of the change in the basin as parameters vary.

A second widely used class of methods for calculation of basins of attraction are cell mapping methods [29]-[33]. These methods avoid the calculation of long time trajectories by dividing the region of investigation into a number of discrete "cells", trajectories from which define a discrete mapping from each cell to another. The cell mappings can be analyzed in various manners to give a full picture of the long time trajectory behavior of the system and hence the basins of attraction. These methods are extremely useful for low dimensional systems. However, the calculation of the mapping from cell to cell does not scale well to dimensions greater than 3 and we wished to use a method which is applicable to larger systems.

A third method of investigation of the basins of attraction is the calculation of stable manifolds of the fixed points and periodic orbits of the system. These manifolds will act as boundaries between basins of attraction. A number of efficient and general numerical methods have been proposed for such calculations; see [34] and references therein. Although these methods generalize theoretically to high dimensional systems, like the cell mapping methods they are not currently practical for dimensions greater than 3 or 4 .

2) Monte Carlo Methods: We may randomly select a finite number of starting points within our region of investigation. We can then use a numerical procedure to calculate the trajectories starting from these points, over a suitably long time interval, and examine whether they lead to an attractor or to collapse. This method has been used with success in [35] to determine a wide variety of the possible dynamics in a dynamical system, some of which were not found by path following methods.

This method has several benefits. It is easy to adjust the accuracy of the method by simply changing the number of initial points, the error tolerance of the integration routine and the time period of the total integration. This allows a preliminary investigation at low tolerance; subsequent calculations can be performed at higher accuracy once regions of interest have been identified. The method is adaptable to systems of higher dimension and of arbitrary structure-we may select an integration 
routine which most efficiently calculates trajectories for that particular problem. The method is simple to code and to use. Although this is a rather brute force approach to basin calculation, the continuing rapid fall in the cost of computational capacity makes this method viable as an experimental tool and perhaps an industrial one also.

Some care must be taken in the choice of integration routine used. The computed trajectories are only estimates of those in the real system, but we desire them to have the same qualitative behavior; that is we wish the numerical schemes, considered as discrete dynamical systems to "shadow" the underlying continuous system. It has been proved that several commonly used integration methods do not have this property [36]. To guarantee proper qualitative results, for DAE systems and stiff systems such as power-system models, we must use methods such as backward differentiation-even though they may be more computationally expensive.

For the results below, integration was initially performed using the RK-2(3) solver with adaptive step-sizing, which is a default method in Matlab. However, the step-sizes used by this explicit method were too small to be efficient for the long time period calculations necessary; in addition the step-size selection algorithm created quite obvious errors and discontinuities, probably related to the stiffness of the system (1). As an alternative, the implicit second order method TR-BDF2 [37] was used with much greater success.

\section{BIFURCATION ANALYSIS OF THE 3-Bus ModEL}

The analysis and methods developed in the last section can now be applied to a full study of the dynamics of the 3-bus model (1). To do this we firstly consider varying separately the two parameters $Q_{1}$ and $P_{1}$, determine the bifurcation points, and then follow paths of these points in both parameters. The resulting calculation allows us to divide up parameter space into regions in which the behavior of the overall system will be qualitatively different. In the next section we give examples of the dynamical behavior in each region.

\section{A. 1-D Bifurcation Diagrams}

1) Varying the Reactive Power Demand: Increasing reactive power demand is believed to be a factor in many voltage collapse incidents; for this reason $Q_{1}$ used here and elsewhere [5] as the primary continuation parameter. The bifurcation diagram of fixed points of the system (1) with respect to $Q_{1}$ is shown in Fig. 2, with fixed $P_{1}=0$.

Here, the upper branch is stable close to the saddle-node bifurcation at $Q_{1}^{\mathrm{SN} 1}=11.41146$ and the lower branch is unstable.

The two saddle-node bifurcations on the unstable branch at $Q_{1}^{\mathrm{SN} 2}=3.92380$ and $Q_{1}^{\mathrm{SN} 3}=0.90944$ have not been previously presented. Previous analysis of power-system models has not paid much attention to the unstable branch of fixed points. However, these points are important; in determining the maximum basin of attraction associated with stable fixed points and when they lie on a homoclinic orbit or are associated with boundary crisis events destroying a strange attractor. Any structure in the unstable branch, such as the two bifurcations, is,

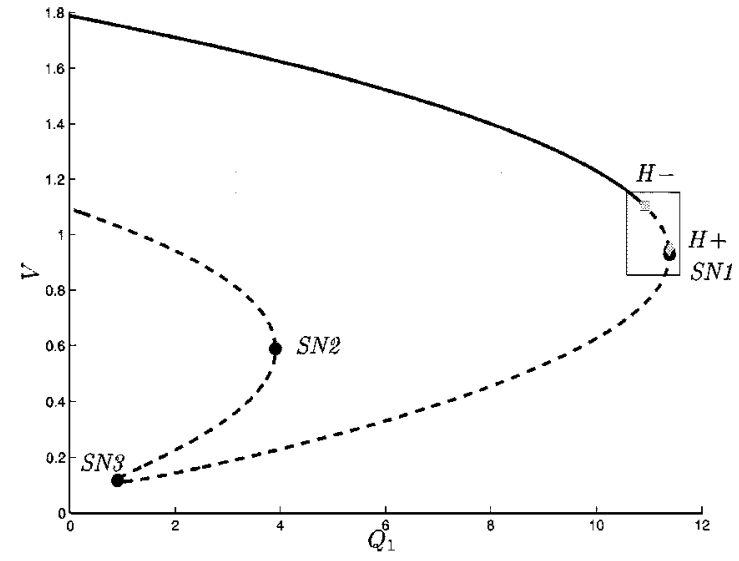

Fig. 2. Bifurcation diagram in $Q_{1}$ for the 3-bus model, $P_{1}=0$.

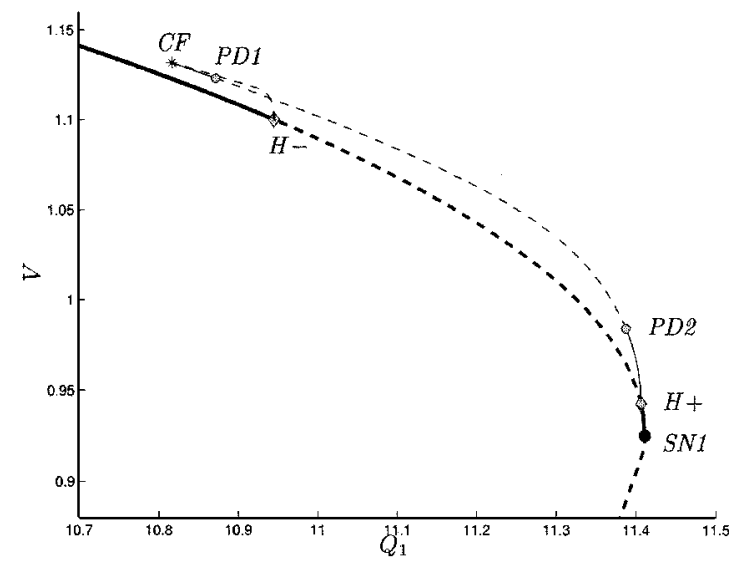

Fig. 3. Bifurcation diagram in $Q_{1}$ for 3-bus model (Detail—periodic orbits).

therefore, of potential interest and so unstable paths must be calculated and considered carefully.

On the branch of stable fixed points exists a pair of Hopf-bifurcation points, at $Q_{1}^{H-}=10.94609$ and $Q_{1}^{H+}=11.40676$. The first of these two points is a subcritical bifurcation where an unstable periodic orbit coalesces with the fixed point (as we increase $Q_{1}$ ). The second is supercritical giving rise to a stable periodic orbit. Paths of periodic orbits bifurcate from the primary branch at each Hopf point (Fig. 3). The unstable periodic orbit bifurcating from $Q_{1}^{H-}$ subsequently coalesces with a stable periodic orbit in a cyclic fold bifurcation at $Q_{1}^{\mathrm{CF}}=10.81766$. This point has a significant effect on the stability of the electrical system. For $Q_{1}<Q_{1}^{\mathrm{CF}}$ the only stable operating point of the 3-bus model is a fixed point, whereas for $Q_{1}>Q_{1}^{\mathrm{CF}}$ the fixed point coexists with a stable periodic orbit. As the periodic orbit has a non trivial domain of attraction, it is quite possible that the 3-bus model of the power system may settle into a stable periodic orbit. This may well not be a safe operating condition, although it is bounded and does not result in a voltage collapse. We show presently that many cyclic folds are associated with the homoclinic orbit which emanates from the BT-point and that they may be computed as parameters vary. If $Q_{1}$ is increased from $Q_{1}^{\mathrm{CF}}$ then the stable periodic orbit undergoes a sequence of period doubling bifurcations, the first being at $Q_{1}^{\mathrm{PD} 1}=10.87327$, and subsequently evolves into a strange attractor. The attractor gives an erratic behavior in the electrical 


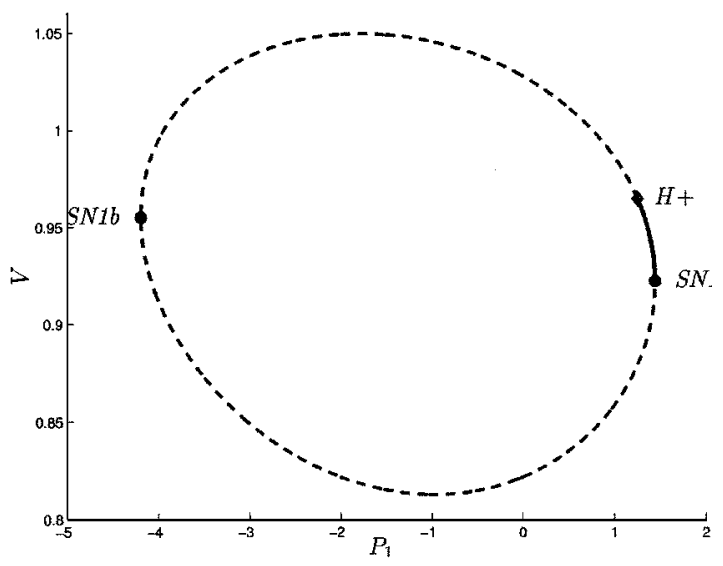

Fig. 4. Bifurcation diagram in $P_{1}$ for 3-bus model, $Q_{1}=11.25$.

system, but the resulting orbits are all bounded and are indeed not very far from the fixed point.

At the point $Q_{1}^{\mathrm{BC}}=10.8943$, it was observed in that the strange attractor disappears in a boundary crisis where it collides with the unstable manifold of the neighboring saddle point [38] (this is also observed in a similar system with different parameters [9]). If $Q_{1}$ is decreased from the value $Q_{1}^{H+}$, the super-critical Hopf bifurcation, then we observe a series of super-critical period doubling bifurcations, leading to a chaotic attractor. This, in turn, vanishes at $Q_{1}=11.3768$.

For $10.8943<Q_{1}<11.3768$, there are no bounded attracting states and most initial conditions lead to voltage collapse.

We present figures of the strange attractor, of periodic orbits and of collapsing trajectories in Section V, in our discussion of the system's $\omega$-limit sets (and their meanings) at different parameter values.

2) Varying the Real Power Demand: To clarify and unfold the structure observed in the above section we now consider varying the real power demand $P_{1}$. Starting from the stable fixed point at $P_{1}=0, Q_{1}=11.25$ we compute the fixed point as a function of $P_{1}$, keeping $Q_{1}$ fixed. This yields the bifurcation diagram presented in Fig. 4. This bifurcation diagram has an approximately circular structure, with saddle-node bifurcations at $P_{1}^{\mathrm{SN} 1}=1.44896$ and $P_{1}^{\mathrm{SN} 2}=-4.18066$. We observe that whereas the bifurcation at $P_{1}^{\mathrm{SN} 1}$ is a coalescence of a stable and an unstable fixed point, that at $P_{1}^{\mathrm{SN} 2}$ is a coalescence of two unstable fixed points. For this fixed value of $Q_{1}$, these two bifurcations give the maximum and minimum real power transfer limits.

As in the previous investigation, we observe a super-critical Hopf bifurcation at $P_{1}^{H+}=1.28383$ leading to the creation of a stable periodic orbit. Again, these orbits exhibit cyclic fold and period doubling bifurcations. We will see presently that further bifurcations from these periodic orbits leads to more complex behavior.

\section{B. 2-D Bifurcation Diagram and BT Point}

We now extend our continuation methods to compute paths in the $\left(P_{1}, Q_{1}\right)$ parameter space of the saddle-node, Hopf, and cyclic fold bifurcation points computed in the previous two subsections, as described in Section III-C. Examples of the ex- tended systems used for continuation of saddle-node and Hopf bifurcations are given in [19] and are implemented in AUTO [16] and other packages. To follow cyclic fold points we use the method as implemented in AUTO [16].

1) Physical Meaning of Parameter Values: These curves naturally all continue into the parameter range $P_{1}<0$. This is apparently an unphysical approach as this would correspond to a scenario in which the loads (or at least the constant part of the empirical induction motor load) are providing power to the rest of the network. However, our aim is to investigate the qualitative behavior of a highly reduced system and demonstrate the effectiveness of certain methods, so there is a two-fold justification for considering the $P_{1}<0$ parameter region.

It is possible that by following paths of certain bifurcation points that we will naturally consider values of the parameters which are not especially physical; for example if $P_{1}<0$. However, by identifying organizing centres (such as the BT-point) for the dynamics in these regimes we can infer a lot about the dynamics in the more physically interesting regions of parameter space. So we may step into the region in which the model is possibly invalid in order to find dynamical phenomena which may have effects in the "valid" region; such a procedure is widely used in other investigations of physical systems. In addition, the position in 2-D parameter space of the structures and phenomena described may be dependent on further parameters. Hence if these parameters were to change or be adjusted, the phenomena occurring in a "physically unrealistic" region could move to a parameter region where they might be observable.

Concisely-we are not troubled by taking $P_{1}<0$ since it may lead us to find things out for $P_{1}>0$ that we would not have found otherwise.

2) 2-D Loci of Saddle-Node Bifurcations: The paths of the main saddle-node bifurcation and the two subsidiary saddle-node bifurcations on the the unstable branch of fixed points are given in Fig. 5. The main fold can be thought of as the maximum reactive power $Q_{1}$ transfer point over all values of $P_{1}$. The 2-D diagram shows that the curve of saddle-node points itself has a maximum value when $Q_{1}=11.45$ occurring when $P_{1}=-1.5$. Observe that this is close to the value obtained when $P_{1}=0$. Thus, the observed maximum reactive power point given when $P_{1}=0$ is in fact close to the point of maximum reactive power transfer over all values of $P_{1}$.

3) 2-D Loci of Hopf Bifurcations: A similar diagram to that of the locus of saddle-node bifurcation points is plotted for the paths of the sub-critical and super-critical Hopf bifurcation points (Fig. 6). It can be seen that for $P_{1}>0$ the two Hopf points approach each other and eventually coalesce at $P_{1} \approx 1.46$. The periodic orbits and chaotic structure in the region between the Hopf points vanishes for $P_{1}>1.5$ leading to relatively simple and predictable dynamics in this region of parameter space. This scenario of the annihilation (or creation) of complex periodic behavior has been observed in many systems, see, for example, [39] and [25].

For $P_{1}<0$, the sub-critical Hopf bifurcation occurs at consistently lower values of $Q_{1}$ as $P_{1}$ is decreased. This implies that the system could be more susceptible to destabilising periodic behavior at these lower real power demand. In contrast, 


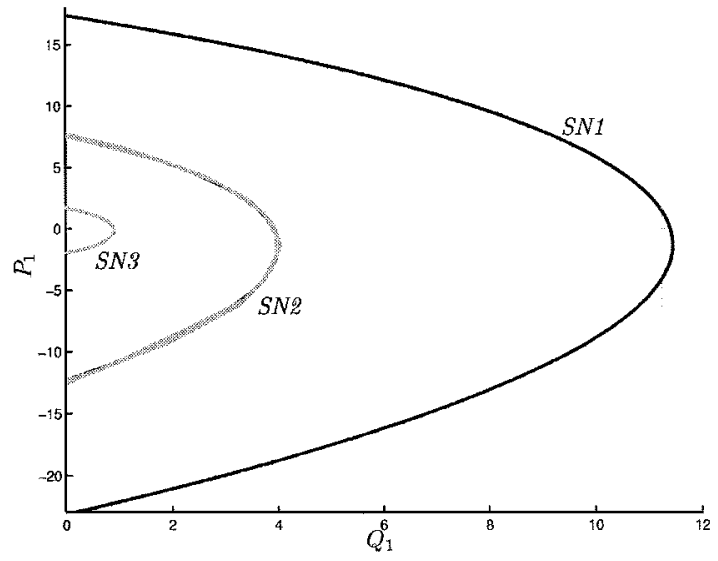

Fig. 5. Paths of saddle-node (fold) bifurcations in 3-bus model, in $P_{1}-Q_{1}$ parameter plane.

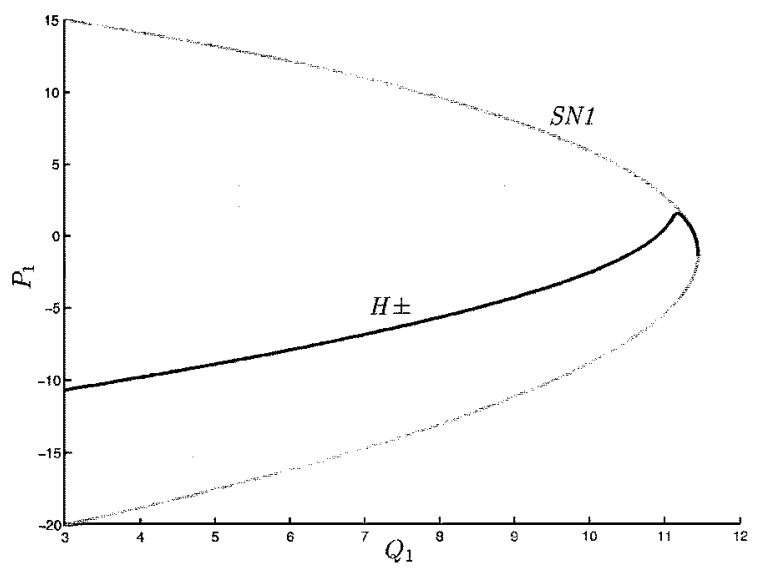

Fig. 6. Path of Hopf bifurcations in 3-bus model, in $P_{1}-Q_{1}$ parameter plane.

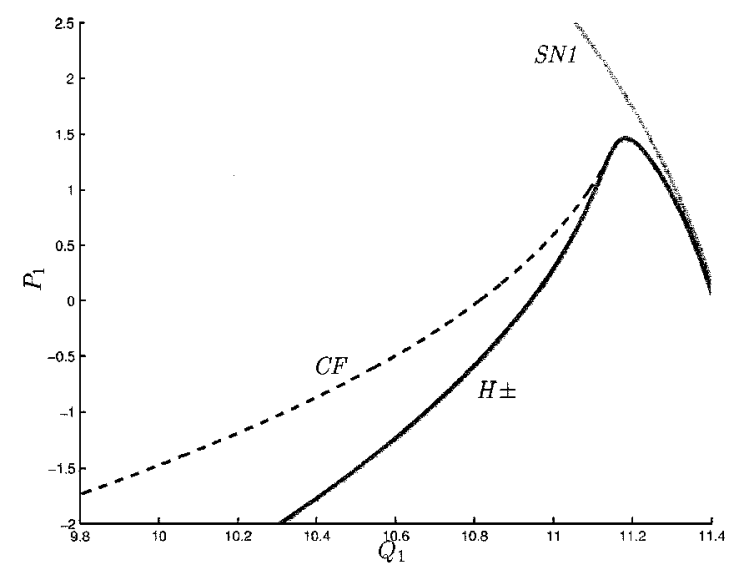

Fig. 7. Path of cyclic folds in 3-bus model, in $P_{1}-Q_{1}$ parameter plane.

the path of the right-hand, supercritical, Hopf bifurcation approaches the path of the main saddle-node tangentially as we decrease $P_{1}$. This can be see more clearly if we plot both paths at once, Fig. 7.

4) 2-D Loci of the Cyclic Fold Points: The branch of cyclic fold points which includes the point observed when $P_{1}=0, Q_{1}=10.81766$ may be followed using the method described above yielding the curve shown in Fig. 7. Observe that this curve exists for values of $Q_{1}$ in general rather less than

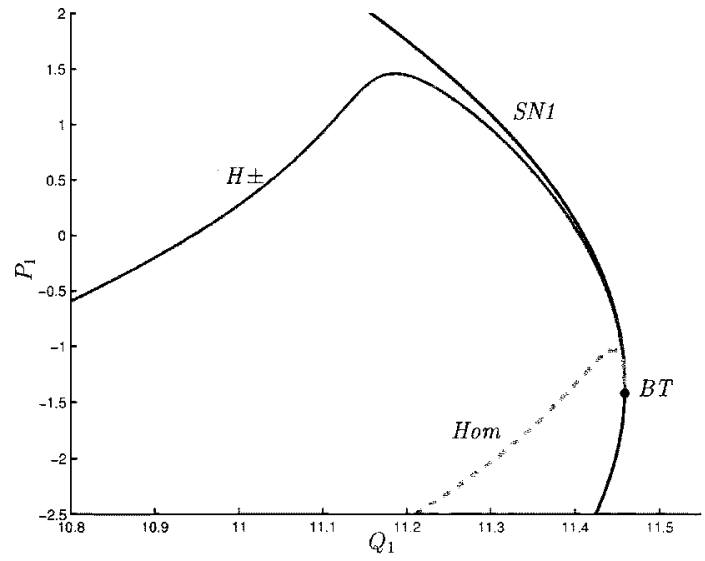

Fig. 8. 2-D bifurcation diagram for 3-bus model, in $P_{1}-Q_{1}$ parameter plane, including BT-point.

the value at which Hopf bifurcation occurs, with a consequent reduction in the parameter range in which we see only a stable fixed point. The locus of the cyclic fold point intersects the locus of the Hopf points at $Q_{1}=11.14798, P_{1}=1.31408$. This curve of cyclic folds is not unique; other curves of cyclic-folds associated with the Šil'nikov bifurcation will be presented in the next section.

5) BT-Point in the 3-Bus Model: The tangential intersection of the paths of saddle-node and Hopf bifurcation points gives strong evidence for the existence of a BT-point as described in the last section. The existence of this point is determined computationally by monitoring the second rightmost eigenvalue of the system Jacobian matrix as we compute the path of saddle-node bifurcations in Fig. 5. This second eigenvalue must vanish at the BT-point; if it changes sign over two successive continuation steps then the BT-point must lie between them. To further refine the location of this point we apply the algorithm described in the Appendix, using one of these two saddle-node bifurcation points as an initial guess.

Applying this method to the 3-Bus system gave a BT-point at $Q_{1}=11.45907, P_{1}=-1.41946,\left(\delta_{m}, \omega_{m}\right.$, $\delta, V)^{T}=(0.42224,0,0.21355,0.93164)^{T}$. The corresponding eigenvalues of the system at this point are $0,0,-3.059$ and -41.955 . Observe that two of these are real and negative and have no effect on the dynamics, merely indicating the rate of convergence onto the centre-manifold of the BT-point. The BT-point now acts as an organizing centre for paths of saddle-node bifurcations, Hopf bifurcations, period doubling bifurcations and homoclinic orbits.

\section{Homoclinic Orbits}

The theory discussed earlier ensures the existence of a path of homoclinic orbits in $\left(P_{1}, Q_{1}\right)$ parameter space starting at the BT-point. Each such homoclinic orbit contains an unstable fixed point.

The method described in Section III-E was tried for (1) but failed in practice, as the initial guess for the homoclinic orbit was not sufficiently precise. An alternative procedure which did work was instead to approximate the path of homoclinic orbits by a path of high period periodic orbits (e.g., period $T^{*}=200$ ). To determine such an orbit, the path of periodic orbits created 


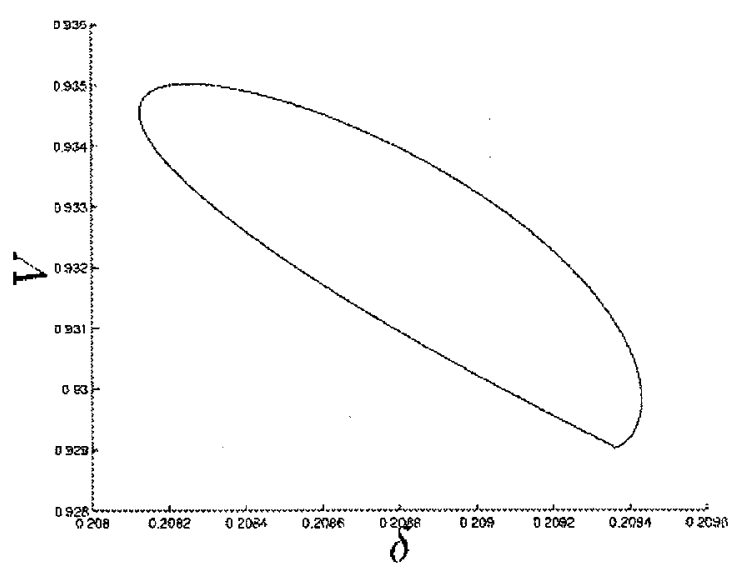

(a)

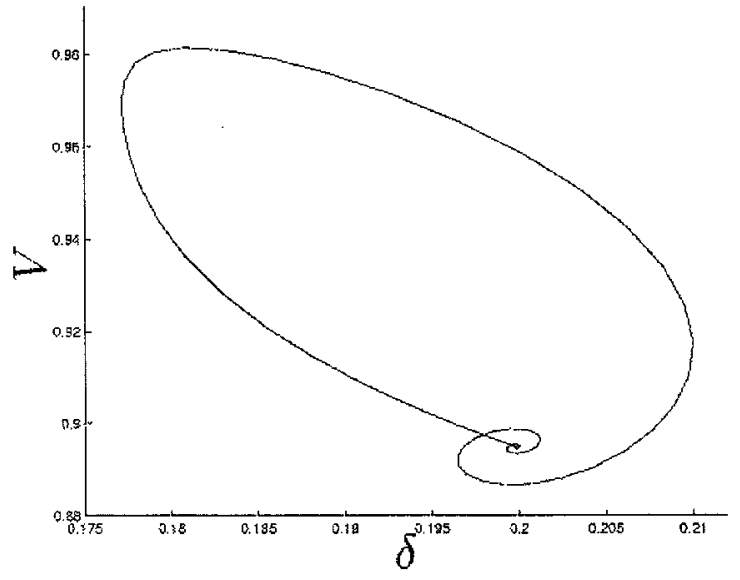

(b)

Fig. 9. Approximate homoclinic orbits. (a) Close to and (b) slightly away from the BT-point.

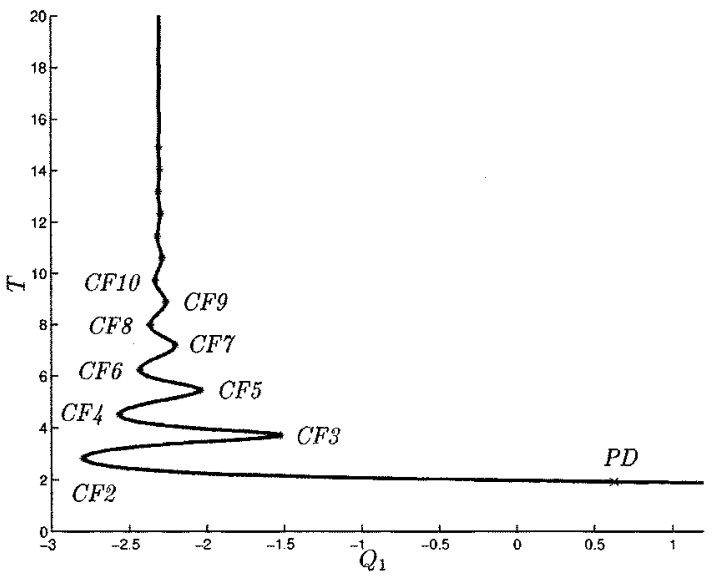

Fig. 10. Time period of period orbits in 3-Bus model, plotted against parameter $P_{1}$. $\left(Q_{1}=11.25\right)$.

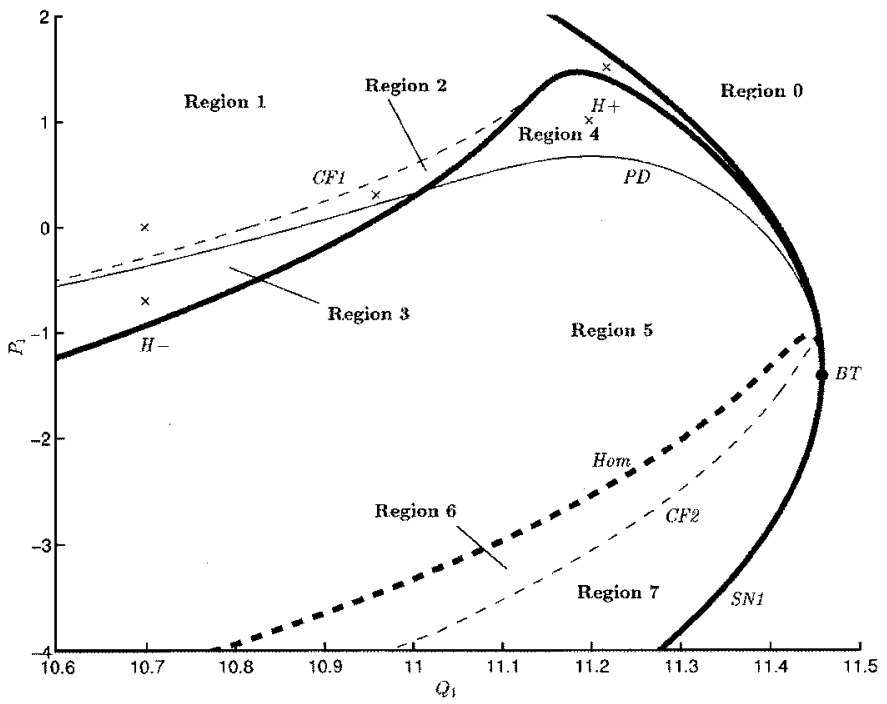

Fig. 11. Full 2-D bifurcation diagram.

in a Hopf bifurcation at $P_{1}=-1.31329$ and $Q_{1}=11.45918$ was followed as $P_{1}$ was varied until the orbit had a period $T^{*}$. The locus of the period $T^{*}$ orbit was then followed as $P_{1}$ and
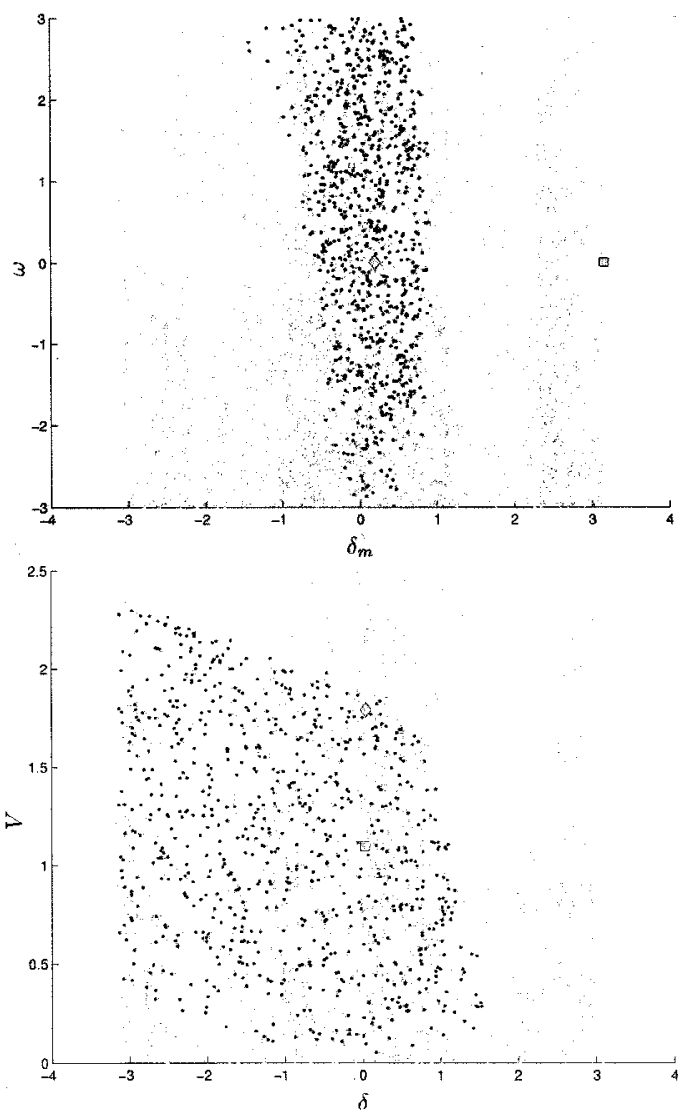

Fig. 12. Basin investigation results, $\left(Q_{1}, P_{1}\right)=(0,0)$.

$Q_{1}$ were varied. The resulting curve in parameter space of these high period points is then shown in Fig. 8.

The resulting computed curve is clearly tangent to the curve of Hopf and Saddle-node points at the BT-point. Furthermore, the curve has a high curvature at this point. This curvature indicates that the normal form will only be an accurate reduction of (1) very close to the BT-point and this explains the difficulties with the first procedure for calculating the homoclinic orbits.

We illustrate the above calculation by giving the trajectory of the resulting high period orbit in the two cases of 

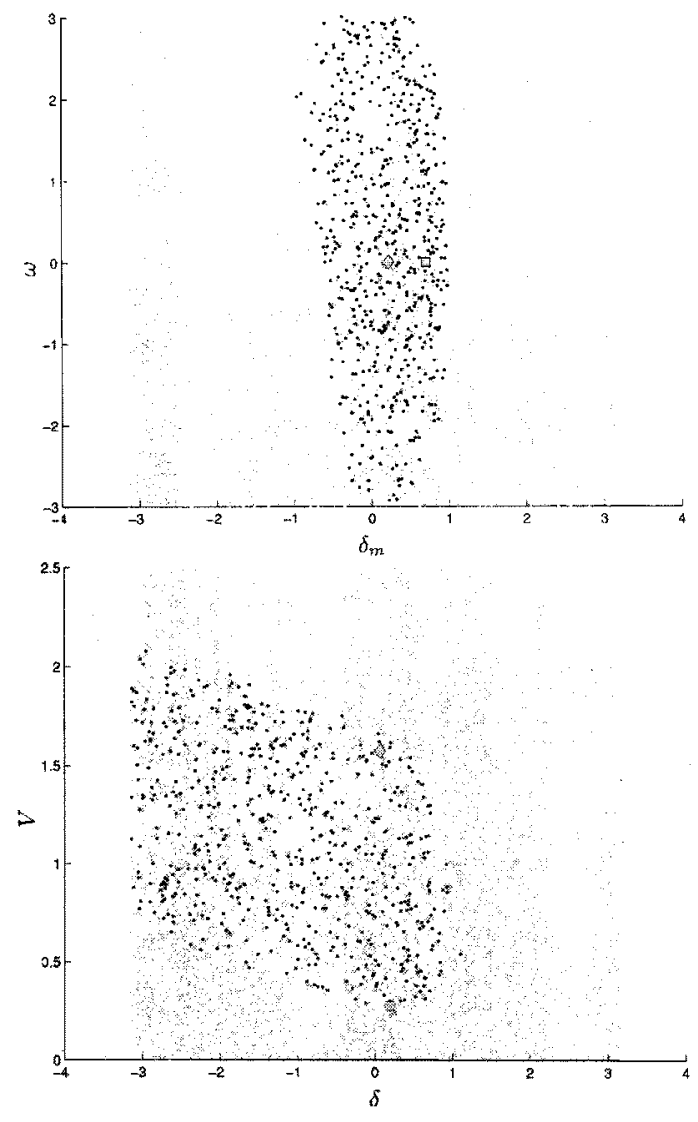

Fig. 13. Basin investigation results, $\left(Q_{1}, P_{1}\right)=(5,0)$.

$\left(Q_{1}, P_{1}\right)=(11.45916,-1.33260)$ which is close to the BT-point and $\left(Q_{1}, P_{1}\right)=(11.43866,-1.05069)$ which is rather more distant (Fig. 9). Observe the change in the qualitative form of the orbit in these two cases.

1) Šil'nikov Homoclinicity: The 3-Bus system contains homoclinic orbits of Šil'nikov type where the homoclinic orbit intersects a fixed point which is a saddle focus (i.e., it has a one dimensional unstable manifold, and its two least stable, stable eigenvectors have complex eigenvalues and span a 2-D space.) These homoclinic orbits are sometimes referred to as "saddle-focus" homoclinics. Assume the linearization around the unstable fixed point contained within the homoclinic orbit has one positive real eigenvalue $\nu_{1}=\alpha>0$ and next rightmost eigenvalues are a complex conjugate pair $\nu_{2,3}=-\beta \pm \gamma i$. We can define a useful quantity ${ }^{2} \delta=\beta / \alpha$; this can be thought of as the ratio of the speed of rotation and the speed of ejection of orbits near the fixed point [40]. The homoclinic orbit is said to be of Šil'nikov type if $\delta<1$. In this case the linearization at the unstable fixed point is dominated by the rightmost, complex, pair of eigenvalues; the stable manifold is then essentially two dimensional, and the homoclinic orbit spirals around the fixed point as it approaches it. In addition there is a characteristic bifurcation diagram for the periodic orbits near this type of homoclinic orbit, and much can also be said about the presence of chaotic orbits [41].

This is well illustrated by considering the 3-Bus model itself. Consider the 1-D bifurcation diagram in $P_{1}$ (Fig. 4) where

\footnotetext{
${ }^{2}$ This ratio is not to be confused with our state variable $\delta$; phase angle at load.
}
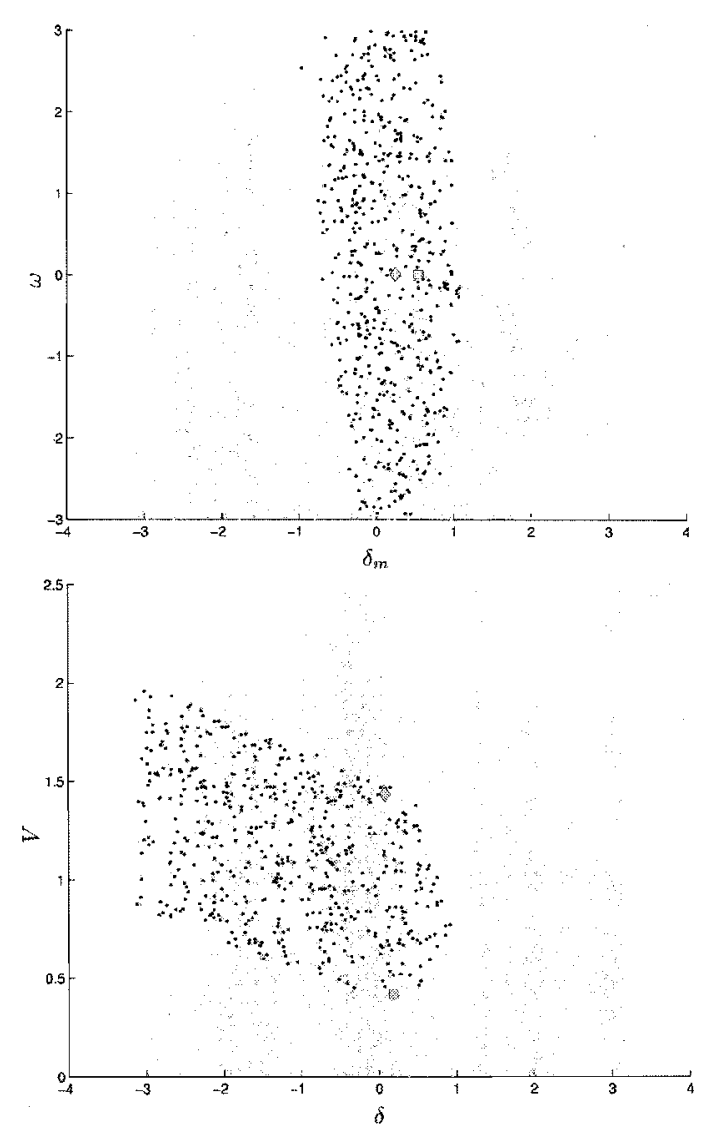

Fig. 14. Basin investigation results, $\left(Q_{1}, P_{1}\right)=(7.5,0)$.

$Q_{1}=11.25$ and calculate the periodic orbits emanating from the Hopf point $P_{1}^{H+}$ ). A plot of the time period of the orbits on this path (Fig. 10) show the period increasing with an oscillatory pattern, with orbits of increasing period converging through multiple cyclic folds $P_{1}^{\mathrm{CF} n}$ toward the homoclinic orbit at $P_{1}^{h}=-2.30456$. This pattern is characteristic of the behavior of a (global) Šil'nikov bifurcation, with a sequence of multiple cyclic-fold points also accumulating onto the homoclinic orbit. It is also possible to follow the periodic orbit created in the period doubling bifurcation at $P_{1}^{\mathrm{PD}}=-0.62682$. This orbit also displays an oscillatory structure as it converges toward a double-pulse homoclinic orbit.

If we examine the eigenvalues of the unstable fixed point at $Q_{1}=11.25, P_{1}^{h}=-2.30456$, we find that $\delta=0.01344$ confirming our observations. The value of $\delta$ changes along the path of the approximate homoclinic orbits, accounting for the qualitative change in the orbits shown in Fig. 9.

The main features of a Šil'nikov bifurcation are: 1) the creation of an infinite set of cyclic fold points; 2) the creation of an infinite set of period-doubling bifurcations either side of each fold; 3 ) the existence of complex chaotic behavior close to the homoclinic orbit. In the latter case, it will be very difficult to resolve which part of this region is liable to lead to a voltage collapse. We can however calculate the path of cyclic folds passing through $<P_{1}^{\mathrm{CF} 1}, Q_{1}=11.25$ which acts as a boundary for this region in the 2-parameter plane.

This particular form of the dynamics, while important, occurs in this example for nonphysical values of the real power demand 


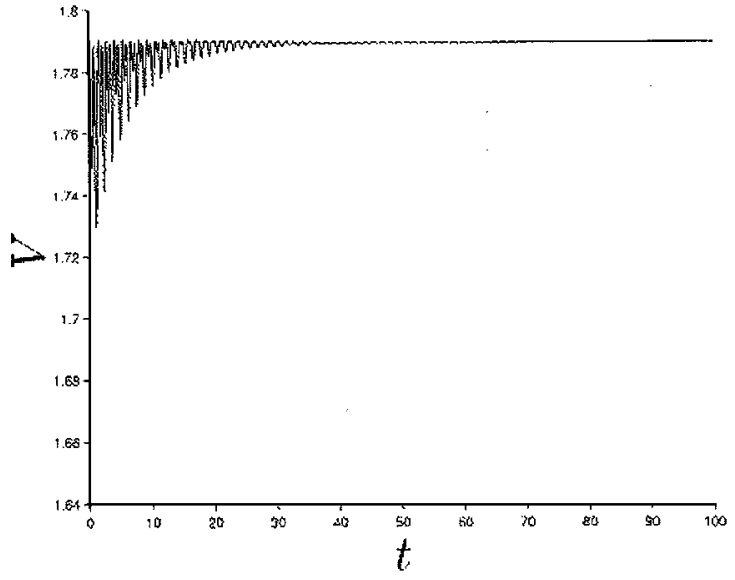

(a)

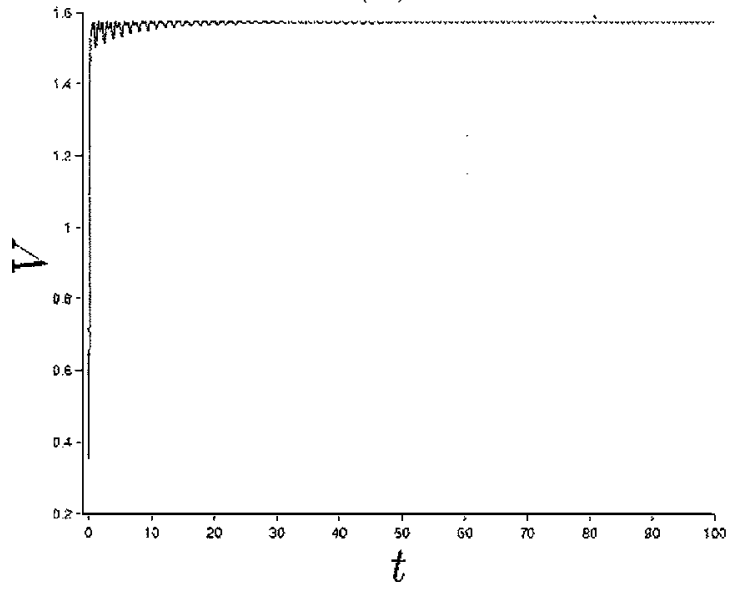

(c)

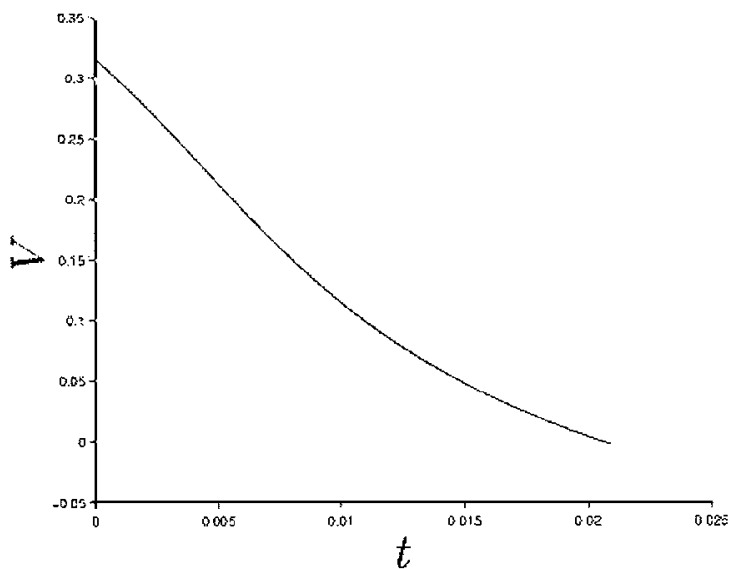

(b)

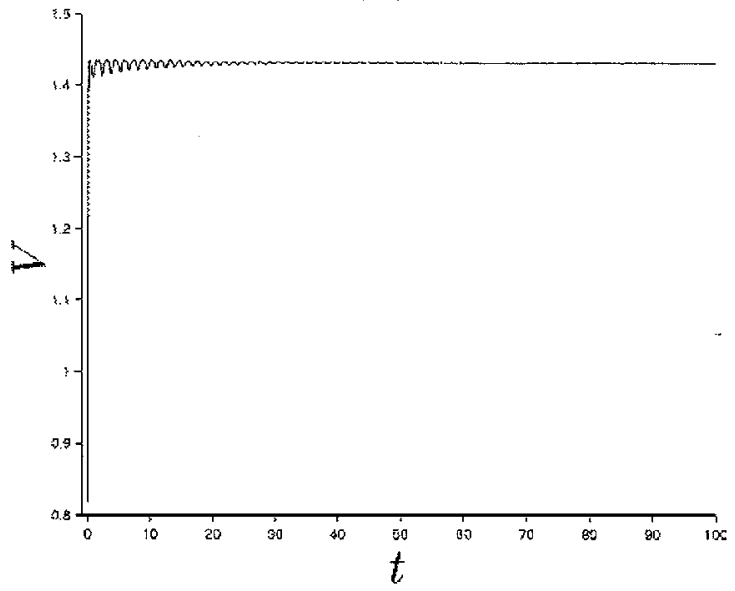

(d)

Fig. 15. Typical trajectories, (a, b) $\left(Q_{1}, P_{1}\right)=(0,0)$, (c) $\left(Q_{1}, P_{1}\right)=(5,0),(\mathrm{d})\left(Q_{1}, P_{1}\right)=(7.5,0)$.

$P_{1}$. However, in contrast, the loci of some of the cyclic fold and period doubling bifurcation points created at this bifurcation can be followed by continuation in $P_{1}$ and $Q_{1}$ into more physically interesting regions of parameter space.

In an exciting new figure (Fig. 11) we plot all these loci. This gives what we believe is a complete picture of the structure of the bifurcations in the two parameter space. This picture is likely to be similar in any related power-system models with a BT-point and an associated Šil'nikov bifurcation.

\section{THE IMPLiCATIONS OF THE BIFURCATION DiAgRAM ON THE SYSTEM DYNAMICS}

The significance of the previous calculations is that we can divide up parameter space into different regions where we expect to see contrasting dynamical behavior. This allows us to estimate which parameter regions are likely to be dangerous from a point of view of voltage collapse and how this might occur.

\section{A. Interpreting the Bifurcation Diagram}

With respect to problems such as voltage collapse, we may consider the usefulness of the bifurcation diagram in two different ways.
One interpretation is to think of the parameters as real, theoretically measurable values which change over time (but over a time scale much larger than that of individual system trajectories at a particular fixed parameter value). So considering all other parameter values as fixed, we would be interested in what happens as we move continuously through parameter space, especially in the case where we are initially at a stable steady state and the experience a bifurcation as our path through parameter space intersects a path of bifurcation points of this fixed point.

Alternatively we can think of the parameters as being fixed and use the bifurcation diagram to explain the behavior of the system at those fixed parameter values, in terms of the dynamical objects (e.g., stable and unstable fixed points, periodic orbits and homoclinic orbits) that the bifurcation diagram tells us may occur at that point. This could be useful if we assume the system at this parameter value to model the behavior of power system after a fault or a disturbance. In this post-fault or post-disturbance case we would not assume that we were at a stable fixed point, but instead we would be interested in what might happen to a general trajectory starting at some point in phase space.

The first interpretation would be useful for real-time monitoring of a system, the second for predicting and analysing the behavior of a proposed system. The two interpretations are not entirely distinct. 

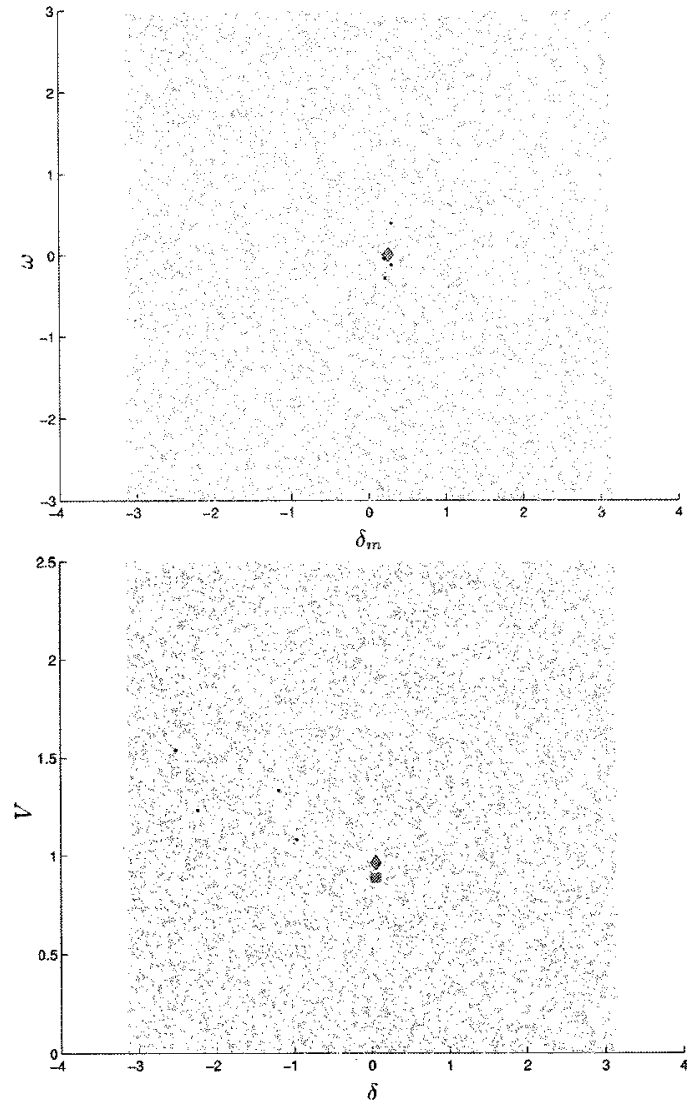

Fig. 16. Basin investigation results, $\left(Q_{1}, P_{1}\right)=(11.22,1.5)$.

\section{B. Parameters for Trajectory and Monte-Carlo Computation}

The following results were each computed in Matlab using the method described in Section III.F.2. 8000 initial conditions were used from the range $\left(\delta_{m}^{0}, \omega^{0}, \delta^{0}, V^{0}\right) \in[-\pi, \pi] \times[-2,2] \times$ $[-\pi, \pi] \times[0,2.5]$. The TR-BDF2 method used a relative tolerance of $10^{-3}$ and an absolute tolerance of $10^{-5}$. Trajectories were said to have escaped (voltage collapse) and integration halted if $\left(\delta_{m}, \omega, \delta, V\right)(t) \notin\left[\delta_{m}^{0}-2 \pi, \delta_{m}^{0}+2 \pi\right] \times[-2.5,2.5] \times$ $\left[\delta^{0}-2 \pi, \delta^{0}+2 \pi\right] \times[0,3.5]$. Here we are not allowing phase changes at load or generator of more than $2 \pi$, or allowing negative voltage magnitude - this would be physically unrealistic. Calculation of trajectories was halted after $100 \mathrm{~s}$ (or after 50 000 steps), at which time the trajectory was assumed to have converged sufficiently close to an attractor.

These parameters constitute a reasonable trade off between accuracy and speed. More initial points, smaller tolerances, longer integration time and a less naive definition of "attracted" would give more accurate results, but the method would be less useful as an experimental tool. In particular it has been noted [9] that in the presence of periodic and chaotic attractors, the time to collapse may be atypically long. Our observations confirm this and so we suggest use of longer integration time as a way of improving the results rather than more points or better tolerances.

\section{Dynamics for Regions in the 2-D Bifurcation Diagram}

We take each region in Fig. 11 in turn and discuss the likely dynamics and their practical implications. Where applicable we include time series plots of typical trajectories.
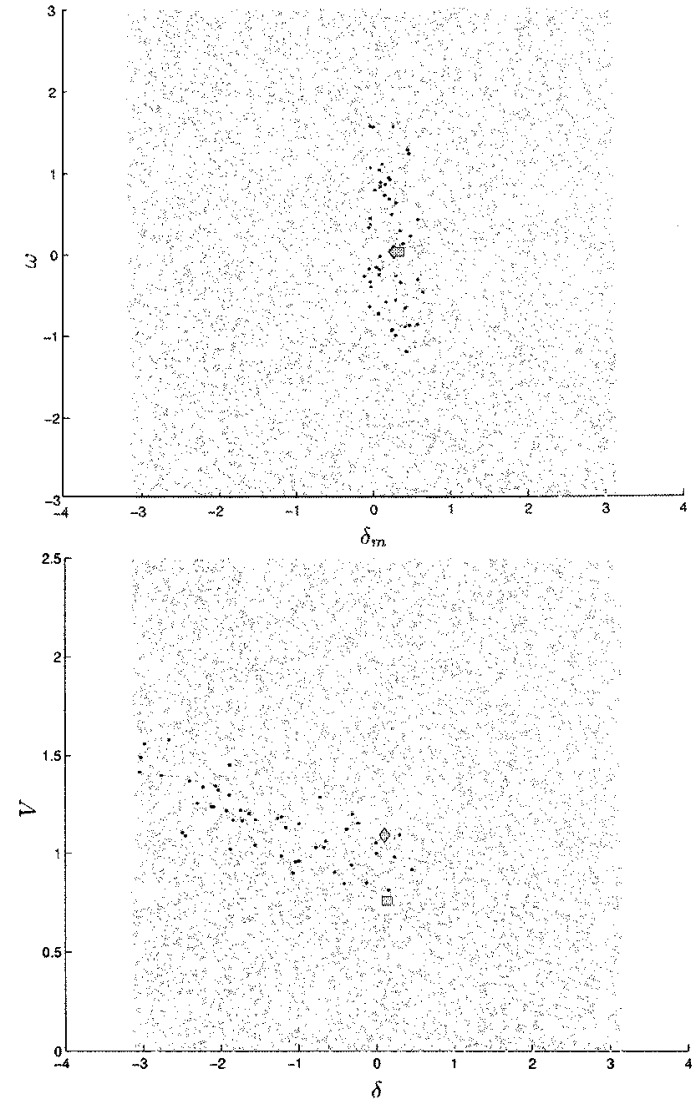

Fig. 17. Basin investigation results, $\left(Q_{1}, P_{1}\right)=(10.96,0.3)$.

1) Region 0: Outside the region bounded by 2-parameter path of the primary saddle-node point. There are no known stable (fixed point or periodic) solutions in this region.

2) Region 1: This region is characterized by reasonably low values of the parameter $Q_{1}$, the existence of a stable steady state at a higher (physically realistic) voltage, and an odd number of unstable steady states at a lower (physically unrealistic) voltage.

Some parameter values in this region (i.e., $P_{1}<0$ ) may be less physically valid than others, but as a whole this parameter region can be thought of as being operationally "safe". The degree of this safety depends on the proximity to the paths of bifurcation points.

For investigation of basins of attraction in this region, we choose four typical parameter values $\left(Q_{1}, P_{1}\right)=$ $(0,0),(5,0),(7.5,0)$ and $(11.22,1.5)$; recall that the primary saddle-node bifurcation occurs at $\left(Q_{1}=11.41146, P_{1}=0\right)$. The results are plotted in Figs. 12-14, and 16 respectively. Recall that the only physically realistic attractor in this parameter region is the stable fixed point. All trajectories not leading to this point diverge to $\infty$ (voltage collapse).

Each shows the randomly chosen initial values $\left(\delta_{m}^{0}, \omega^{0}, \delta^{0}, V^{0}\right)$ projected onto the planes $\left(\delta_{m} \omega\right)$ (plane of generator variables) and $(\delta, V)$ (plane of load variables). The values are color coded depending on whether they lead to the stable fixed point (black) or lead to collapse (grey). We also plot on each graph the stable (diamond) and unstable (square) fixed points for that parameter value.

The first three of these figures show that there is a clear reduction of in the size of the basin of attraction as the reactive power 


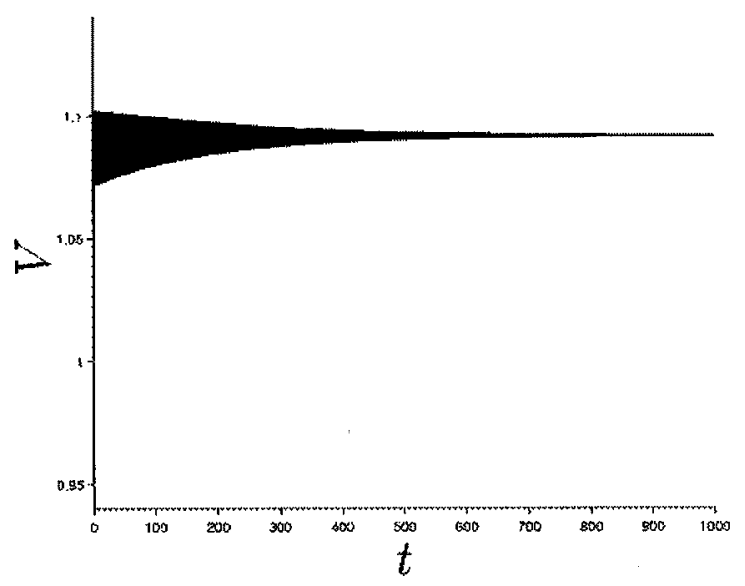

(a)

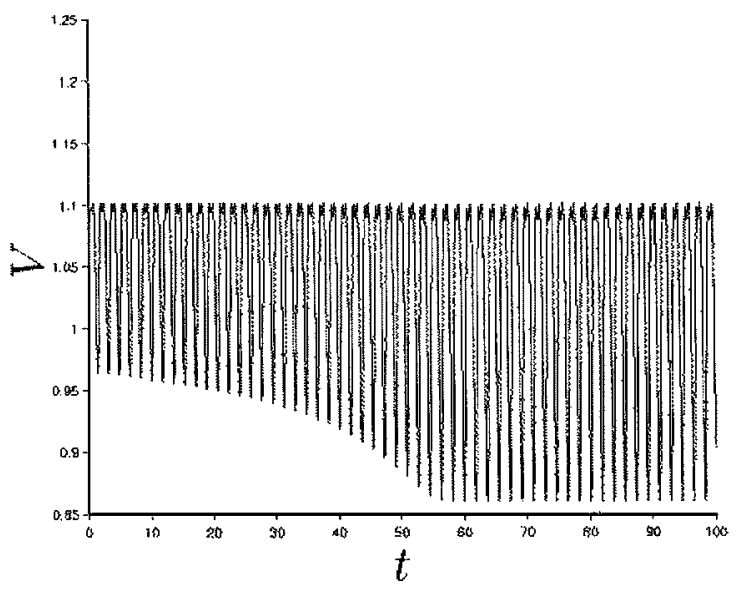

(b)

Fig. 18. Typical trajectories at $\left(Q_{1}, P_{1}\right)=(5,0)$.: (a) attracted to fixed point and (b) attracted to periodic orbit.

increases toward the value at which Hopf and saddle-node bifurcations occur (but still remains some distance from paths of such points). We may also see that the basin boundary suggested by these computations does not have the hyper-spherical structure as might be suggested by some Lyapunov functions. Instead, there are points inside the basin of attraction (especially in the $\delta$ direction) which lie a long way from the stable fixed point.

In Fig. 15 we plot some typical attracted and collapsing trajectories at these three parameter values.

Fig. 16 shows the basin computations at a point $\left(Q_{1}, P_{1}\right)=$ $(11.22,1.5)$ close to both the path of Hopf bifurcations and the path of saddle-node points. There are very few points attracted to the stable fixed point. The closeness of this parameter value to the bifurcation points means that the basin of attraction for the operating point has all but disappeared.

3) Region 2: The region is bounded by a path of cyclic folds CF1 (i.e., limit points on paths of periodic orbits), a path of subcritical Hopf bifurcations $H-$, and a path of period doublings PD.

In this region, there exist multiple stable states-a stable steady state and a stable periodic orbit. There are also unstable periodic orbits and unstable fixed points. If a disturbance were to occur, it would be possible for the trajectory to be attracted to the stable periodic orbit. The resulting oscillatory behavior may lead to some form of collapse or shutdown.

We plot the basin computation for the parameter value $\left(Q_{1}, P_{1}\right)=(10.96,0.3)$ in Fig. 17. Some sample trajectories at this parameter value, Fig. 18 clearly show attraction to both the stable fixed point and to the stable periodic orbit, depending on the initial condition.

4) Region 3: This region bounded by the path of Hopf bifurcations $H \pm$ and period doublings. PD There are stable periodic orbits and unstable fixed points in this region, but no stable fixed points. The absence of period doublings in this region excludes the possibility of cascades to chaotic behavior. Basin computations for this region, Fig. 19 show that the basin of attraction of the periodic orbits is likely to be extremely small.

We plot a typical periodic orbit in Fig. 20.
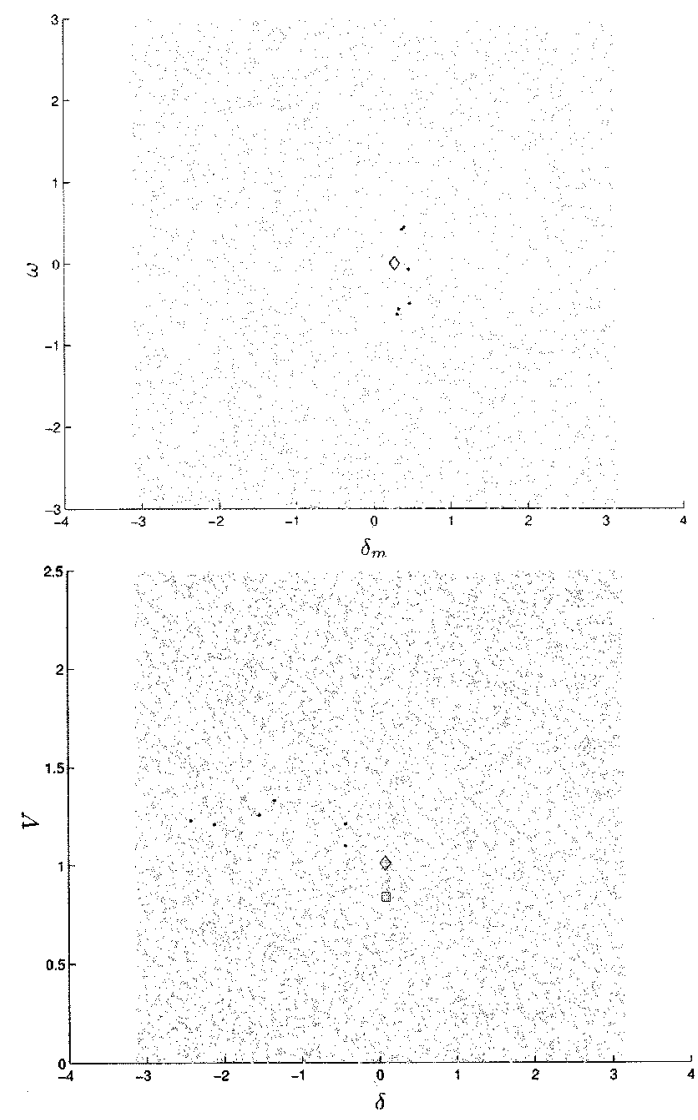

Fig. 19. Basin investigation results, $\left(Q_{1}, P_{1}\right)=(11.2,1.0)$.

5) Region 4: A path of subcritical Hopf bifurcations $H-$ and a path of period doubling bifurcations PD bound the points in this region.

Both stable fixed points and stable (period doubled) periodic orbits are present at some parameter values in this region. (The period doubling path is of period doublings of the stable periodics emanating from the cyclic fold $\mathrm{CF} 1$, not from the subcritical Hopf points $H-$ ). In some parts of this region there may 


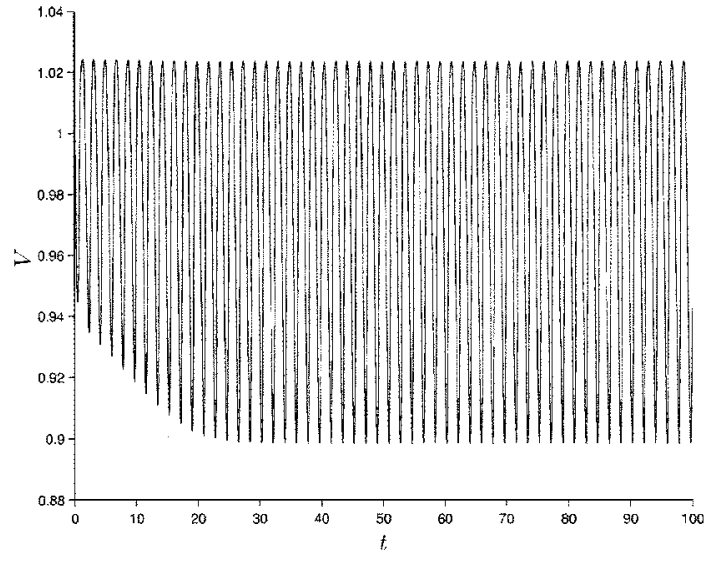

Fig. 20. Periodic Orbit, $\left(Q_{1}, P_{1}\right)=(11.2,1.0)$.
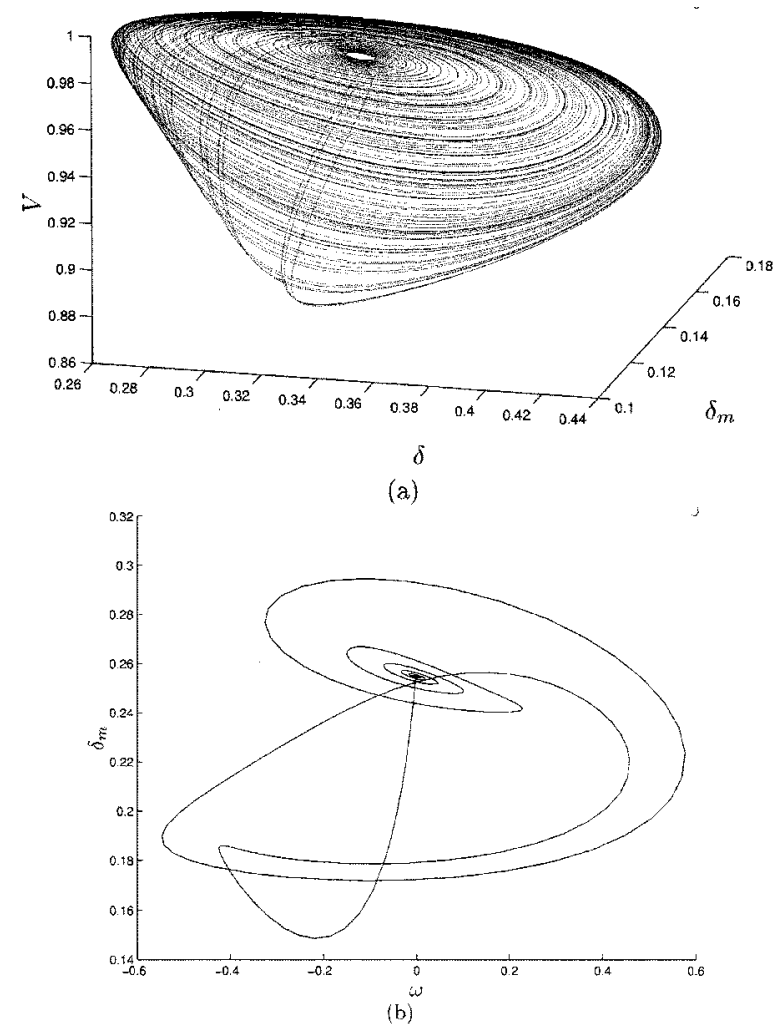

Fig. 21. (a) Strange attractor, $\left(Q_{1}, P_{1}\right)=(11.3768,0.0)$, (b) Double pulse homoclinic, $\left(Q_{1}, P_{1}\right)=(11.25,-1.731537)$.

exist further period doublings and cascades of such leading to chaos. There also exist boundary crises of periodic orbits, also leading to loss of stability.

6) Region 5: No stable fixed points exist in this region, since we have greater values of $Q_{1}$ than at the path $H-$ of subcritical Hopf points.

This region will contain all manner of periodic orbits (stable and unstable), chaotic orbits, possible boundary crises and double pulse homoclinic orbits. Fig. 21 shows a typical strange attractor at the parameter value $\left(Q_{1}, P_{1}\right)=(11.3768,0.0)$ and an approximate double pulse homoclinic orbit at $\left(Q_{1}, P_{1}\right)=(11.25,-1.731537)$. We would expect voltage collapse to be the dominant behavior in this parameter region.
Since much of this region lies well into the "physically realistic" $P_{1}>0$ region, we would want to avoid it as an operating region.

7) Region 6: This region lies between the path of homoclinic orbits $\mathrm{H}$ om emanating from the BT-point and the path of the first cyclic folds CF2 relating to the Sil'nikov behavior. It contains an infinite number of periodic orbits and no stable fixed points.

8) Region 7: There is no known stable behavior or any periodic behavior (unstable or homoclinic) in this region.

\section{CONCLUSION}

We have demonstrated that the dynamics of a simple powersystem model may be investigated with reference to a co-dimension 2-BT point. This acts as an organizing centre for the 2-parameter paths of saddle-node, Hopf and homoclinic bifurcations. The homoclinic orbits can be of Šil'nikov type, leading to multiple periodic and chaotic orbits.

This bifurcation information can then be used to guide an fuller investigation of the 2-parameter dynamics of the model; allowing us to predict the dynamics occurring in different regions. We have shown that significant reductions in the size of basins of attractions of stable fixed points may occur well before actual bifurcation takes place.

We have discussed, in general terms, the numerical methods used for this analysis, allowing for its application to larger problems. The power-system models clearly have a rich dynamical structure; investigation of this is of interest to both mathematician and power engineer. We suggest that further investigation, along similar lines, of larger (hence more physically realistic) systems is both practically possible and highly useful.

\section{APPENDIX I}

\section{NUMERICAL DETECTION OF BT-POINTS}

In order to numerically detect and accurately locate BT-points we must first be able to compute 2-parameter paths of folds (or Hopf-bifurcations). This may be achieved by defining a suitable system, roots of which correspond to folds (or Hopf points). We can then apply a continuation method to this system.

While approximating such a path of co-dimension 1 bifurcation points in the two-parameter plane, we may check at each iteration whether we are in the neighborhood of a BT point. For instance, while following a path of saddle-node bifurcations we might monitor whether the eigenvalue with the second smallest magnitude changes sign in its real part. Having detected the possibility of a BT point we can then apply an algorithm to locate the BT point to desired accuracy. Once again, this is by the solution of a suitable extended system, roots of which define a BT point. Such a system is

$$
F(x, \lambda, \mu, v, w)=\left[\begin{array}{c}
f \\
f_{x} v \\
c_{o}^{T} v-1 \\
f_{x} w-v \\
c_{0}^{T} w
\end{array}\right]=0
$$


Here, we are saying that we require a solution to the generalized eigenvalue problem $f_{x} v=0, f_{x} w=v$. In practice, this formulation of the BT-point detection problem is highly inefficient. Given that our dynamical system (3) has dimension $n$, the Jacobian matrix for system (5) (which must be inverted in a Newton-type solution method) is of dimension $(3 n+2)$ by $(3 n+2)$. A more efficient decomposition of this system, based on methods proposed by [24] and [42], is now presented.

\section{A. Decomposition}

Lemma 1: Define

$$
A=\left[\begin{array}{cc}
f_{x} & b_{0} \\
c_{o}^{T} & 0
\end{array}\right]
$$

where $b_{0}, c_{0} \in \mathbb{R}^{n}$. Then $A$ is nonsingular if (for instance) $b_{0}=$ $f_{\lambda}$ or $f_{\mu}$, and $c_{0} \notin \mathcal{N}\left(f_{x}\right)$.

Proof: ABCD-lemma.

Theorem 2: (Griewank and Reddien [42], as presented in Beyn [24])

Let $A$ be defined as above and let functions $g, h:\left(\Omega \times \mathbb{R}^{2}\right) \rightarrow$ $\mathbb{R}$ and vectors $v, w \in \mathbb{R}^{n}$ be defined by the solutions to

$$
A\left[\begin{array}{l}
v \\
g
\end{array}\right]=\left[\begin{array}{l}
0 \\
1
\end{array}\right] \text { and } A\left[\begin{array}{l}
w \\
h
\end{array}\right]=\left[\begin{array}{l}
v \\
0
\end{array}\right]
$$

Then, roots $x^{0}, \lambda^{0}, \mu^{0}$ of the system

$$
S(x, \lambda, \mu)=\left[\begin{array}{l}
f(x, \lambda, \mu) \\
g(x, \lambda, \mu) \\
h(x, \lambda, \mu)
\end{array}\right]=0
$$

also satisfy the generalized right eigenvalue problem given in (5), and so define BT-points.

In addition, if we define $\bar{g}, \bar{h}:\left(\Omega \times \mathbb{R}^{2}\right) \rightarrow \mathbb{R}$ and vectors $\psi, \zeta \in \mathbb{R}^{n}$ by

$$
\left[\psi^{T}, \bar{g}\right] A=[0,1] \quad \text { and } \quad\left[\zeta^{T}, \bar{h}\right] A=[\psi, 0]
$$

then

$$
\begin{aligned}
g=\bar{g} & =-\psi^{T} f_{x} v \\
h=\bar{h} & =\psi^{T} v \\
g_{z} & =-\psi^{T} f_{x z} v \\
h_{z} & =-\psi^{T} f_{x z} w-\zeta^{T} f_{x z} v
\end{aligned}
$$

where $z$ can be either $x, \lambda$ or $\mu$; the roots of (8) and solutions of (9) give

$$
\begin{array}{ll}
\psi^{T} f_{x}=0, & \psi^{T} b_{0}=1 \\
\zeta^{T} f_{x}=\psi, & \zeta^{T} b_{0}=0
\end{array}
$$

that is to say the generalized left eigenvalue problem for $f_{x}$.

Proof: See [42].

\section{B. Newton's Method}

Newton-type methods to solve (8) require us to solve the four $(n+1)$-dimensional linear systems in (7) and (9) (involving inversion of $A$ and $\left.A^{T}\right)$ and then solve the $(n+2)$-dimensional system

$$
\left[\begin{array}{lll}
f_{x} & f_{\lambda} & f_{\mu} \\
g_{x} & g_{\lambda} & g_{\mu} \\
h_{x} & h_{\lambda} & h_{\mu}
\end{array}\right]\left[\begin{array}{l}
\delta x \\
\delta \lambda \\
\delta \mu
\end{array}\right]=-\left[\begin{array}{l}
f \\
g \\
h
\end{array}\right]
$$

where we use (10) to give the derivatives of $g$ and $h\left(f_{x x}, f_{x \lambda}, f_{x \mu}\right.$ can be calculated explicitly or numerically). The following theorem presents an efficient way to solve this system, first shown in [42], but presented here in a slightly different form and with a minor correction.

Theorem 3: If we find $g, h, v, w, \psi$ and $\zeta$ from (7) and (9) as above and solve the following three linear systems in $A$

$$
\begin{gathered}
A\left[\begin{array}{c}
\tilde{f}_{\lambda} \\
\left.\left(\psi^{T} f_{\lambda}\right)+\left(g c_{0}^{T} \tilde{f}_{\lambda}\right)\right]
\end{array}\right]=\left[\begin{array}{c}
f_{\lambda} \\
0
\end{array}\right], \\
A\left[\begin{array}{c}
\tilde{f}_{\mu} \\
\left(\psi^{T} f_{\mu}\right)+\left(g c_{0}^{T} \tilde{f}_{\mu}\right)
\end{array}\right]=\left[\begin{array}{c}
f_{\mu} \\
0
\end{array}\right], \\
A\left[\begin{array}{c}
\tilde{f} \\
\left(\psi^{T} f\right)+\left(g c_{0}^{T} \tilde{f}\right)
\end{array}\right]=\left[\begin{array}{c}
f \\
0
\end{array}\right],
\end{gathered}
$$

then, the Newton correction $(\delta x, \delta \lambda, \delta \mu)^{T}$ in (12) is given by solution of the $(3 \times 3)$ system

$$
\left[\begin{array}{ccc}
g & \psi^{T} f_{\lambda} & \psi^{T} f_{\mu} \\
g_{x} v & g_{\lambda}-g_{x} \tilde{f}_{\lambda} & g_{\mu}-g_{x} \tilde{f}_{\mu} \\
h_{x} v & h_{\lambda}-h_{x} \tilde{f}_{\lambda} & h_{\mu}-h_{x} \tilde{f}_{\mu}
\end{array}\right]\left[\begin{array}{c}
\delta t \\
\delta \lambda \\
\delta \mu
\end{array}\right]=-\left[\begin{array}{c}
\psi^{T} f \\
g-g_{x} \tilde{f} \\
h-h_{x} \tilde{f}
\end{array}\right]
$$

and

$\delta x=(v \delta t)-\left(\tilde{f}_{\lambda} \delta \lambda\right)-\left(\tilde{f}_{\mu} \delta \mu\right)-\tilde{f}$

Proof: Add a further two rows and columns to (12) along with two dummy variables $\delta \phi, \delta t \in \mathbb{R}$ to get

$$
\left[\begin{array}{ccccc}
f_{x} & b_{0} & 0 & f_{\lambda} & f_{\mu} \\
c_{o}^{T} & 0 & -1 & 0 & 0 \\
0 & -1 & 0 & 0 & 0 \\
g_{x} & 0 & 0 & g_{\lambda} & g_{\mu} \\
h_{x} & 0 & 0 & h_{\lambda} & h_{\mu}
\end{array}\right]\left[\begin{array}{c}
\delta x \\
\delta \phi \\
\delta t \\
\delta \lambda \\
\delta \mu
\end{array}\right]=-\left[\begin{array}{l}
f \\
0 \\
0 \\
g \\
h
\end{array}\right] .
$$

(Note that the principal sub-matrix of this expanded matrix is $A$ and that $\delta \phi=0$ ). The first two "rows" of (16) can be rearranged to give

$$
\begin{aligned}
{\left[\begin{array}{cc}
f_{x} & b_{0} \\
c_{o}^{T} & 0
\end{array}\right]\left[\begin{array}{l}
\delta x \\
\delta \phi
\end{array}\right]=\delta t } & {\left[\begin{array}{l}
0 \\
1
\end{array}\right] } \\
& -\delta \lambda\left[\begin{array}{c}
f_{\lambda} \\
0
\end{array}\right]-\delta \mu\left[\begin{array}{c}
f_{\mu} \\
0
\end{array}\right]-\left[\begin{array}{l}
f \\
0
\end{array}\right]
\end{aligned}
$$

Pre-multiplying this by $A^{-1}$ we get

$$
\begin{aligned}
{\left[\begin{array}{l}
\delta x \\
\delta \phi
\end{array}\right]=\delta t A^{-1}\left[\begin{array}{l}
0 \\
1
\end{array}\right]-\delta \lambda A^{-1}\left[\begin{array}{c}
f_{\lambda} \\
0
\end{array}\right] } \\
-\delta \mu A^{-1}\left[\begin{array}{c}
f_{\mu} \\
0
\end{array}\right]-A^{-1}\left[\begin{array}{l}
f \\
0
\end{array}\right]
\end{aligned}
$$


We know from (7) that $A^{-1}\left[\begin{array}{l}0 \\ 1\end{array}\right]=\left[\begin{array}{l}v \\ g\end{array}\right]$. The other three terms involving $A^{-1}$ are found as follows. Consider systems of the form

$$
A\left[\begin{array}{l}
\alpha \\
\beta
\end{array}\right]=\left[\begin{array}{l}
\gamma \\
0
\end{array}\right]
$$

where $\alpha, \gamma \in \mathbb{R}^{n}$ and $\beta \in \mathbb{R}$. Pre-multiplying this by $\left[\psi^{T}, 0\right]$, gives

$$
\psi^{T} f_{x} \alpha+\beta\left(\psi^{T} b_{0}\right)=\psi^{T} \gamma
$$

From (9) we have $\psi^{T} f_{x}+g c_{0}=0$ and $\psi^{T} b_{0}=1$. Hence $\beta=\psi^{T} \gamma+g c_{o}^{T} \alpha$. We can let $\gamma$ be successively $f_{\lambda}, f_{\mu}$ and $f$; for these three cases let $\alpha$ be referred to as $\tilde{f}_{\lambda}, \tilde{f}_{\mu}$ and $\tilde{f}$ respectively, giving the triple linear system (13).

Equation (18) now gives us (15) and also

$$
0=\delta \phi=(g \delta t)-\left(\psi^{T} f_{\lambda} \delta \lambda\right)-\left(\psi^{T} f_{\mu} \delta \mu\right)-\psi^{T} f .
$$

This gives the first row of (14); the second and third rows are given by substituting (15) into the last two rows of (16).

\section{Summary}

This decomposition of the BT-point detection problem gives us a number of smaller systems to solve (when compared with solving (5) directly), involving just 2 large matrices ( $A$ and $A^{T}$ ) plus a $3 \times 3$ system.

In addition, we can use $\psi, \zeta$ and (10) as part of efficient branch switching algorithms in order to initiate the computation of paths of homoclinic orbits and Hopf bifurcations in the neighborhood of the BT point. Further details of this can be found in [24].

\section{REFERENCES}

[1] K. Walve, "Modeling of power-system components at severe disturbances," in Proc. Int. Conf. Large High Voltage Electric Systems, Aug. 1986. CIGRÉ paper 38-18.

[2] U.S. Department of Energy, "The Electric Power Outages in the Western United States July 2-3, 1996," U.S. Department of Energy, Washington, D.C., Report to the President, Aug. 1996.

[3] F. Bourgin, G. Testud, B. Heilbronn, and J. Verseille, "Present practices and trends on the french power system to prevent voltage collapse," IEEE Trans. Power Systems, vol. 8, pp. 778-788, Aug. 1993.

[4] I. Dobson, H. D. Chiang, J. S. Thorp, and L. F. Ahmed, "A model of voltage collapse in electric power systems," in Proc. IEEE 27th Conf. Decision and Control, Dec. 1988, pp. 2104-2109.

[5] I. Dobson and H. D. Chiang, "Toward a theory of voltage collapse in electric-power systems," Syst. Control Lett., vol. 13, pp. 253-262, 1989.

[6] E. H. Abed, A. M. A. Hamdan, and H.-C. Lee, "On bifurcations in power-system models and voltage collapse," in Proc. IEEE 29th Conf. Decision and Control, 1990, pp. 3014-3015.

[7] V. Ajjarapu and B. Lee, "Bifurcation-theory and its application to nonlinear dynamic phenomena in an electrical-power system," IEEE Trans. Power Systems, vol. 7, pp. 424-431, Feb. 1992.

[8] H. D. Chiang, C. W. Liu, P. P. Varaiya, F. F. Wu, and M. G. Lauby, "Chaos in a simple power system," IEEE Trans. Power Systems, vol. 8, pp. 1407-1417, Nov. 1993

[9] H. O. Wang, E. H. Abed, and A. M. A. Hamdan, "Bifurcations, chaos, and crises in voltage collapse of a model power system," IEEE Trans. Circuits Syst. I, vol. 41, pp. 294-302, Apr. 1994.

[10] A. H. Nayfeh, A. M. Harb, and C. M. Chin, "Bifurcations in a powersystem model," Int. J. Bifurcation Chaos, vol. 6, no. 3, pp. 497-512, 1996.
[11] C. W. Tan, M. Varghese, P. Varaiya, and F. F. Wu, "Bifurcation, chaos, and voltage collapse in power-systems," Proc. IEEE, vol. 83, pp. 1484-1496, Nov. 1995.

[12] P. Glendinning, Stability, Instability and Chaos, Cambridge Texts in Applied Mathematics. Cambridge, MA: Cambridge Univ. Press, 1994.

[13] J. Guckenheimer and P. Holmes, Nonlinear Oscillations, Dynamical Systems and Bifurcations of Vector Fields, Number 42 in Applied Mathematical Sciences. New York: Springer-Verlag, 1983.

[14] P. Kundur, Power System Stability and Control. New York: McGrawHill, 1984.

[15] C. W. Groetsch, Elements of Applicable Functional Analysis. New York: Marcel Dekker, 1980.

[16] E. J. Doedel, A. R. Champneys, T. F. Fairgrieve, Y. A. Kuznetsov, B. Sandsted, and X. Wang, AUTO 97-Continuation and Bifurcation Software for Ordinary Differential Equations (With HomCont). Montreal, Canada: Concordia Univ., 1997.

[17] H. D. Chiang, T. P. Conneen, and A. J. Flueck, "Bifurcations and chaos in electric power systems: Numerical studies," J. Franklin Inst., vol. 331B, no. 6, pp. 1001-1036, 1994.

[18] A. Jepson and A. Spence, "Folds in solutions of two parameter systems and their calculation, part I," SIAM J. Numer. Anal., vol. 22, no. 2, pp. 347-368, 1985.

[19] B. De Dier, D. Roose, and P. Van Rompay, "Interaction between fold and hopf curves leads to new bifurcation phenomena," J. Computat. Appl. Math., vol. 26, no. 1, pp. 171-186, 1989.

[20] D. Roose and B. De Dier, "Numerical determination of an emanating branch of Hopf bifurcation points in a two-parameter problem," SIAM J. Scientific Stat. Comput., vol. 10, no. 4, pp. 671-685, 1989.

[21] E. Freire, A. J. Rodriguez-Luis, E. Gamero, and E. Ponce, "A case study for homclinic chaos in an autonomous electronic circuit-A trip from Takens-Bogdanov to Hopf-Šil'nikov," Physica D, vol. 62, no. 2, pp. 230-253, 1993.

[22] A. Gragnani, S. Rinaldi, and G. Feichtinger, "Cyclic dynamics in romantic relationships," Int. J. Bifurcation Chaos, vol. 7, no. 11, pp. 2611-2619, 1997.

[23] J. Carr, Applications of Centre Manifold Theory. New York: SpringerVerlag, 1981

[24] W. J. Beyn, "Numerical-analysis of homoclinic orbits emanating from a Takens-Bogdanov point," IMA J. Nume. Anal., vol. 14, no. 2, pp. 381-410, 1994.

[25] J. M. T. Thompson, "Global dynamics of driven oscillators: Fractal basins and indeterminate bifurcations," in Non-Linear Maths \& Applications, P. J. Aston, Ed. Cambridge, U.K.: Cambridge Univ. Press, 1996.

[26] Y. J. Cao, Q. H. Wu, and S. J. Cheng, "An improved Lyapunov function for power-system stability,” Int. J. Control, vol. 65, no. 5, pp. 791-802, 1997.

[27] R. J. Davy and I. A. Hiskens, "Lyapunov functions for multimachine power systems with dynamic loads," IEEE Trans. Circuits Syst. I, vol. 44, pp. 796-812, Sept. 1997.

[28] D. J. Hill, I. A. Hiskens, and I. M. Y. Mareels, "Stability theory of differential/algebraic models of power systems," in Proc. Sādhanā-Academy Engineering Sciences, vol. 18, 1993, pp. 731-747.

[29] C. S. Hsu, "A theory of cell-to-cell mapping dynamical-systems," ASME J. Applied Mech. Trans., vol. 47, no. 3, pp. 931-939, 1980.

[30] C. S. Hsu and R. S. Guttalu, "An unravelling algorithm for global analysis of dynamical systems-An application of cell-to-cell mappings," ASME J. Appl. Mech. Trans., vol. 47, no. 2, pp. 940-948, 1980.

[31] C. S. Hsu, "A generalized theory of cell-to-cell mapping for nonlinear dynamical-systems," ASME J. Appl. Mech. Trans., vol. 48, no. 2, pp. 634-642, 1981.

[32] B. H. Tongue and K. Gu, "Adaptive mesh strategies for interpolated mapping procedures," Int. J. Eng. Sci., vol. 27, pp. 1143-1154, 1989.

[33] J. Levitas and T. Weller, "Poincare linear interpolated cell mapping-Method for global analysis of oscillating-systems," ASME J. Appl. Mech. Trans., vol. 62, pp. 489-495, 1995.

[34] B. Krauskopf and H. Osinga, "Globalizing two-dimensional unstable manifolds of maps," Int. J. Bifurcation Chaos, vol. 8, no. 3, pp. 483-503, 1998.

[35] C. J. Budd and A. G. Lee, "Double impact orbits of periodically forced impact oscillators," in Proc. Royal Soc. London Series A, vol. 452, 1996, pp. $2719-2750$.

[36] A. M. Stuart and A. R. Humphries, Dynamical Systems and Numerical Analysis. Cambridge, U.K.: Cambridge Univ.Press, 1996.

[37] L. F. Shampine and M. W. Reichelt, "The Matlab ODE suite," SIAM J. Sci. Comput., vol. 18, no. 1, pp. 1-22, 1997.

[38] H. Osinga, private communication, 1998. 
[39] J. M. T. Thompson and F. A. McRobie, "Intermediate bifurcations and the dynamics of driven oscillators," in Proc. 1st Eur. Nonlinear Oscillations Conf., 1993, pp. 107-128.

[40] T. Mullin, Ed., The Nature of Chaos. Oxford, U.K.: Oxford Scientific, 1993.

[41] P. Glendinning and C. Sparrow, "Local and global behavior near homoclinic orbits," J. Stat. Phys., vol. 35, no. 5/6, pp. 645-696, 1984.

[42] A. Griewank and G. W. Reddien, "Computation of cusp singularities for operator equations and their discretizations," J. Comput.Appl. Math., vol. 26, no. 1, pp. 133-153, 1989.

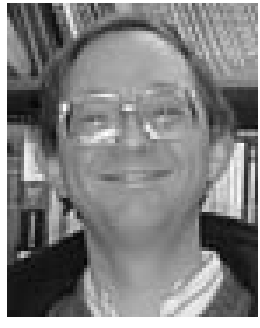

Chris Budd received the M.A. degree from Cambridge Universty, Cambridge, U.K., in 1982, and the D.Phil. degree from Oxford University, Oxford, U.K., in 1986 where he was a research fellow funded by the Central Electricity Generating Board.

He has been Professor of Applied Mathematics at the University of Bath, Bath, U.K., since 1995, where he has developed interests in industrial applied mathematics and numerical analysis and is Co-Director of the Interdisciplinary Centre for Nonlinear Mechanics. He is a keen popularizer of mathematics and its applications and has recently been elected Chair of Mathematics at the Royal Institution.

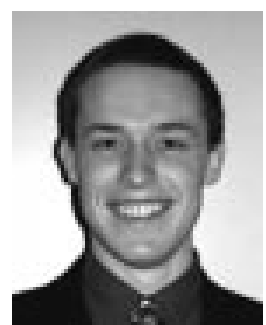

Jon Wilson received the B.Sc. degree in mathematics from the University of Bristol, Bristol, U.K., in 1996, and the M.Sc. and P.D. degrees in the numerical solution of differential equations from the University of Bath, Bath, U.K., which was funded by the EPSRC and the National Grid Company plc, both in 2001.

Dr. Wilson is currently the Director of Net South-West Ltd., Bath, U.K., an IT consultancy specializing in Unix and Internet services. 